\title{
Argyres-Douglas theories, S-duality and AGT correspondence
}

\author{
Takuya Kimura, ${ }^{a}$ Takahiro Nishinaka, $^{a}$ Yuji Sugawara ${ }^{a}$ and Takahiro Uetoko ${ }^{b}$ \\ ${ }^{a}$ Department of Physical Sciences, College of Science and Engineering, Ritsumeikan University, \\ Shiga 525-857\%, Japan \\ ${ }^{b}$ Center for Gravitational Physics, Yukawa Institute for Theoretical Physics, Kyoto University, \\ Kyoto 606-8502, Japan \\ E-mail: rp0047ir@ed.ritsumei.ac.jp, nishinak@fc.ritsumei.ac.jp, \\ ysugawa@se.ritsumei.ac.jp, takahiro.uetoko@yukawa.kyoto-u.ac.jp
}

ABSTRACT: We propose a Nekrasov-type formula for the instanton partition functions of four-dimensional $\mathcal{N}=2 \mathrm{U}(2)$ gauge theories coupled to $\left(A_{1}, D_{2 n}\right)$ Argyres-Douglas theories. This is carried out by extending the generalized AGT correspondence to the case of $\mathrm{U}(2)$ gauge group, which requires us to define irregular states of the direct sum of Virasoro and Heisenberg algebras. Using our formula, one can evaluate the contribution of the $\left(A_{1}, D_{2 n}\right)$ theory at each fixed point on the $\mathrm{U}(2)$ instanton moduli space. As an application, we evaluate the instanton partition function of the $\left(A_{3}, A_{3}\right)$ theory to find it in a peculiar relation to that of $\mathrm{SU}(2)$ gauge theory with four fundamental flavors. From this relation, we read off how the S-duality group acts on the UV gauge coupling of the $\left(A_{3}, A_{3}\right)$ theory.

Keywords: Extended Supersymmetry, Supersymmetric Gauge Theory, Supersymmetry and Duality, Conformal Field Theory

ArXiv EPrint: 2012.14099 


\section{Contents}

1 Introduction 1

2 Generalized AGT correspondence $\quad 4$

3 Irregular states in $\boldsymbol{V}$ ir $\oplus \boldsymbol{H}$ modules $\quad 7$

$\begin{array}{lll}3.1 & \mathrm{U}(2) & \text { version of the original AGT }\end{array}$

$\begin{array}{lll}3.2 & \mathrm{U}(2) & \text { version of the generalized AGT }\end{array}$

4 Nekrasov-type formula for AD matter $\quad 10$

4.1 Decomposition 10

4.2 Identification of $\mathcal{Z}_{Y_{1}, Y_{2}}^{\left(A_{1}, D_{2 n}\right)} \quad 11$

$\begin{array}{lll}4.3 & \text { Identification of } \Lambda & 14\end{array}$

4.4 Consistency check 14

5 Application to $\left(A_{3}, A_{3}\right)$ theory 15

5.1 Prepotential 16

$\begin{array}{lll}5.2 & \text { S-duality } & 18\end{array}$

$\begin{array}{lll}5.3 & \text { Peculiarity of } T & 21\end{array}$

5.4 Turning on couplings, masses and VEVs 22

6 Conclusions and discussions $\quad 24$

$\begin{array}{ll}\text { A Formulae for Nekrasov partition functions } & 27\end{array}$

$\begin{array}{ll}\text { B Orthogonal basis } & 27\end{array}$

$\begin{array}{ll}\text { C Prepotential with massive deformations } & 29\end{array}$

\section{Introduction}

The Nekrasov partition function of four-dimensional $\mathcal{N}=2$ gauge theories with Lagrangian description can often be exactly evaluated by supersymmetric localization [1-5]. The result is written as a sum over fixed points of a torus action on the instanton moduli space. For instance, for $\mathrm{U}(2)$ gauge theory with $N_{f}$ fundamental hypermultiplets, the partition function is evaluated as

$$
\mathcal{Z}_{\mathrm{U}(2)}^{N_{f}}=\mathcal{Z}_{\text {pert }} \sum_{Y_{1}, Y_{2}} \Lambda^{b_{0}\left(\left|Y_{1}\right|+\left|Y_{2}\right|\right)} \mathcal{Z}_{Y_{1}, Y_{2}}^{\mathrm{vec}}(a) \prod_{i=1}^{N_{f}} \mathcal{Z}_{Y_{1}, Y_{2}}^{\text {fund }}\left(a, m_{i}\right),
$$




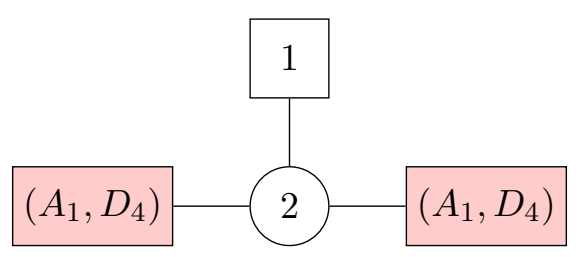

Figure 1. The quiver diagram of the $\left(A_{3}, A_{3}\right)$ AD theory. The left and right boxes stand for two copies of $\left(A_{1}, D_{4}\right)$ theory while the top box stands for a fundamental hypermultiplet of SU(2). The middle circle stands for $\mathrm{SU}(2)$ gauge group coupled to these "matters".

where $b_{0} \equiv 4-N_{f}, \Lambda$ is a dynamical scale, $a$ is the vacuum expectation value (VEV) of a scalar, $Y_{k}$ are Young diagrams, and $\left|Y_{k}\right|$ is the number of boxes in $Y_{k}$. The sum over $\left(Y_{1}, Y_{2}\right)$ can be regarded as a sum over fixed points on the moduli space of $\mathrm{U}(2)$ instantons, and $\mathcal{Z}_{Y_{1}, Y_{2}}^{\text {vec }}$ and $\mathcal{Z}_{Y_{1}, Y_{2}}^{\text {fund }}$ are respectively the contributions of the gauge and matter sectors at these fixed points. The prefactor, $\mathcal{Z}_{\text {pert }}$, is the perturbative contribution that makes the power series in $\Lambda$ start with 1 .

Despite the above success for Lagrangian theories, there is a rich class of fourdimensional $\mathcal{N}=2$ gauge theories whose partition functions are still to be evaluated. These theories involve strongly-coupled CFTs in their matter sector, and therefore their partition functions cannot be directly evaluated by supersymmetric localization. Among other theories, conformal gauge theories coupled to Argyres-Douglas (AD) theories are of particular importance in this class, since they provide a new class of $\mathcal{N}=2$ S-dualities [6-13]. We call such conformal gauge theories "conformally gauged AD theories." One of the simplest examples of such theories is the $\left(A_{3}, A_{3}\right)$ theory described by the quiver diagram in figure 1 , where the $\left(A_{1}, D_{4}\right)$ theory is a particular AD theory. ${ }^{1}$ The beta function of the gauge coupling vanishes here since the contributions of the gauge and matter sectors are exactly canceled.

While the partition functions of conformally gauged AD theories have not been evaluated, there exists a series of non-conformally gauged $\mathrm{AD}$ theories whose partition functions were evaluated via a generalization $[17,18]$ of the AGT correspondence $[19,20]$ (See [21-30] for recent developments on this generalization). In particular, for the theory described by the quiver in figure 2, the partition function was evaluated as the inner product of so-called "irregular states" of Virasoro algebra. The application of the generalized AGT correspondence is possible here since these theories can be engineered by compactifying the $6 \mathrm{~d}(2,0)$ $A_{1}$ theory on a Riemann surface.

The purpose of this paper is to propose a way to compute the partition function of the conformally gauged AD theory in figure 1, using the generalized AGT correspondence for the non-conformally gauged AD theory in figure 2. Since the former has no known construction from the $6 \mathrm{~d}(2,0) A_{1}$ theory, one cannot directly apply the AGT correspondence to it. ${ }^{2}$ Instead, our strategy is to apply the generalized AGT correspondence to the

\footnotetext{
${ }^{1}$ This $\mathrm{AD}$ theory is also called $H_{2}$ theory, $D_{2}(\mathrm{SU}(3))$ theory, and $\left(A_{2}, A_{2}\right)$ theory in the literature. The first series of papers on $\mathrm{AD}$ theories are [14-16].

${ }^{2}$ The theory shown in figure 1 can be constructed by compactifying the $6 \mathrm{~d}(2,0) A_{2}$ or $A_{3}$ theory on a Riemann surface [31,32]. This suggests a possibility of studying its partition function via the higher-rank generalization of the AGT correspondence [33]. Going in this direction would, however, be non-trivial and involved since the $2 \mathrm{~d}$ side is now a higher-rank Toda theory. In this paper, we take a different route.
} 


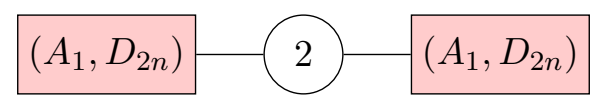

Figure 2. A typical example of gauge theories studied in the generalized AGT correspondence, where $n$ is a positive integer. The circle stands for SU(2) gauge group, and each box stands for an $\left(A_{1}, D_{2 n}\right)$ theory. The $\mathrm{SU}(2)$ vector multiplet is diagonally gauging an $\mathrm{SU}(2)$ sub-group of the $\left(A_{1}, D_{2 n}\right)$ theories.

latter (with a small but crucial modification discussed below) and decompose the resulting partition function as a sum over Young diagrams, as in (1.1). While the theory has a strongly-coupled matter sector, such a decomposition is expected, since the gauge sector of the theory is still described by Lagrangian and its path integral is expected to lead to a sum over fixed points on the instanton moduli space. Once such a decomposition is obtained, one can read off the contribution of the $\left(A_{1}, D_{2 n}\right)$ theory at each fixed point, say $\mathcal{Z}_{Y_{1}, Y_{2}}^{\left(A_{1}, D_{2 n}\right)}$. It is then straightforward to compute the partition function of the conformally gauged AD theory shown in figure 1.

One important point in the above discussion is that the decomposition of the form (1.1) is for $\mathrm{U}(2)$ gauge group while the generalized AGT correspondence is for $\mathrm{SU}(2)$ gauge group. Indeed, for fixed points on the instanton moduli space to be labeled by $\left(Y_{1}, Y_{2}\right)$, the gauge group must be $\mathrm{U}(2)$ instead of $\mathrm{SU}(2)$. Therefore, to decompose the resulting partition function as a sum over $\left(Y_{1}, Y_{2}\right)$, we need a $\mathrm{U}(2)$ version of the generalized AGT correspondence. For the original AGT correspondence, such a $\mathrm{U}(2)$ version is realized by considering the direct sum of Virasoro and Heisenberg algebras (Vir $\oplus H$ ) on the twodimensional side [34]. In this paper, we extend it to the generalized AGT correspondence, by considering irregular states of $\operatorname{Vir} \oplus H$.

Given the U(2) version of the generalized AGT correspondence, one can easily decompose the partition function of $\mathrm{U}(2)$ gauge theory coupled to two $\left(A_{1}, D_{2 n}\right)$ as

$$
\mathcal{Z}_{\mathrm{U}(2)}^{2 \times\left(A_{1}, D_{2 n}\right)}=\mathcal{Z}_{\text {pert }} \sum_{Y_{1}, Y_{2}} \Lambda^{b_{0}\left(\left|Y_{1}\right|+\left|Y_{2}\right|\right)} \mathcal{Z}_{Y_{1}, Y_{2}}^{\mathrm{vec}}(a) \mathcal{Z}_{Y_{1}, Y_{2}}^{\left(A_{1}, D_{2 n}\right)}(a, m, \boldsymbol{d}, \boldsymbol{u}) \widetilde{\mathcal{Z}}_{Y_{1}, Y_{2}}^{\left(A_{1}, D_{2 n}\right)}(a, \widetilde{m}, \tilde{\boldsymbol{d}}, \widetilde{\boldsymbol{u}})
$$

where $m, \boldsymbol{d}=\left(d_{1}, \cdots, d_{n-1}\right)$ and $\boldsymbol{u}=\left(u_{1}, \cdots, u_{n-1}\right)$ are respectively the mass, relevant couplings and VEVs of Coulomb branch operators of the $\left(A_{1}, D_{2 n}\right)$ theory, and $b_{0} \equiv 2 / n$ is the coefficient of the one-loop $\beta$ function. We interpret the sum over $\left(Y_{1}, Y_{2}\right)$ as a sum over fixed points on the moduli space of $\mathrm{U}(2)$ instantons, and identify $\mathcal{Z}_{Y_{1}, Y_{2}}^{\left(A_{1}, D_{2 n}\right)}$ and $\widetilde{\mathcal{Z}}_{Y_{1}, Y_{2}}^{\left(A_{1}, D_{2 n}\right)}$ as contributions from the $\left(A_{1}, D_{2 n}\right)$ theories at each fixed point. The difference between $\mathcal{Z}_{Y_{1}, Y_{2}}^{\left(A_{1}, D_{2 n}\right)}$ and $\widetilde{\mathcal{Z}}_{Y_{1}, Y_{2}}^{\left(A_{1}, D_{2 n}\right)}$ is interpreted as coming from how the $\mathrm{U}(1)$ part of the gauge group is coupled to $\left(A_{1}, D_{2 n}\right)$.

With the above identification of $\mathcal{Z}_{Y_{1}, Y_{2}}^{\left(A_{1}, D_{2 n}\right)}$, we evaluate the partition function of the $\left(A_{3}, A_{3}\right)$ theory as follows. We start with the quiver description shown in figure 1 . When the gauge group in the quiver is $\mathrm{U}(2)$, the partition function is evaluated as

$$
\mathcal{Z}_{\mathrm{U}(2)}=\mathcal{Z}_{\text {pert }} \sum_{Y_{1}, Y_{2}} q^{\left|Y_{1}\right|+\left|Y_{2}\right|} \mathcal{Z}_{Y_{1}, Y_{2}}^{\mathrm{vec}}(a) \mathcal{Z}_{Y_{1}, Y_{2}}^{\text {fund }}(a, M) \prod_{i=1}^{2} \mathcal{Z}_{Y_{1}, Y_{2}}^{\left(A_{1}, D_{4}\right)}\left(a, m_{i}, d_{i}, u_{i}\right),
$$


where $q$ is the exponential of the marginal gauge coupling, and $M$ is the mass of the hypermultiplet. The prefactor $\mathcal{Z}_{\text {pert }}$ is again the perturbative contribution that makes the power series in $q$ start with 1 . Since $\mathcal{Z}_{Y_{1}, Y_{2}}^{\left(A_{1}, D_{2 n}\right)}$ is already read off from the decomposition (1.2), one can explicitly compute the above partition function. When the gauge group is $\mathrm{SU}(2)$, the partition function differs from (1.3) by the contribution of the U(1)-part of the gauge group. Indeed, according to a general discussion in [19], the partition function for $\mathrm{SU}(2)$ gauge group is expected to be given by

$$
\mathcal{Z}_{\mathrm{SU}(2)}=\frac{\mathcal{Z}_{\mathrm{U}(2)}}{\mathcal{Z}_{\mathrm{U}(1)}}
$$

where $\mathcal{Z}_{\mathrm{U}(1)}$ is the partition function of the $\mathrm{U}(1)$ part, and called " $\mathrm{U}(1)$-factor." We use (1.3) and (1.4) to show in particular that the S-duality of the $\left(A_{3}, A_{3}\right)$ theory is in a peculiar relation to that of $\mathrm{SU}(2)$ gauge theory with 4 fundamental flavors. It is an interesting open problem to see how this peculiar relation is connected to a similar relation between the Schur index of the same pair of theories discussed in [35].

The organization of the rest of this paper is the following. In section 2, we briefly review the AGT correspondence and its generalization to $\mathrm{AD}$ theories. In section 3, we consider a U(2)-version of the generalized AGT correspondence, in terms of irregular states of $\operatorname{Vir} \oplus H$. In section 4, we derive a formula for $\mathcal{Z}_{Y_{1}, Y_{2}}^{\left(A_{1}, D_{2 n}\right)}$ corresponding to the gauged $\left(A_{1}, D_{2 n}\right)$ theory, using the $\mathrm{U}(2)$-version of the generalized AGT correspondence discussed in the previous section. In section 5 , we evaluate the partition function of $\left(A_{3}, A_{3}\right)$ theory using our formula for $\mathcal{Z}_{Y_{1}, Y_{2}}^{\left(A_{1}, D_{4}\right)}$. We particularly discuss the S-duality of the theory in connection to the S-duality of $\mathrm{SU}(2)$ gauge theory with four flavors. In section 6 , we conclude and discuss future directions. There are several appendices. Section A includes Nekrasov's formulae for Lagrangian sectors. Section B contains the first few examples of states in a special basis of the highest weight module of $V \operatorname{ir} \oplus H$ that we will use in section 4 . In section $\mathrm{C}$, we explain how the prepotential of the $\left(A_{3}, A_{3}\right)$ theory is constrained by the invariance under (5.45) when all the massive deformations are turned on.

\section{Generalized AGT correspondence}

In this section, we briefly review the AGT correspondence $[19,20]$ and its generalization $[17,18]$. Suppose that $\mathcal{T}_{\mathcal{C}}$ is a four-dimensional $\mathcal{N}=2$ superconformal field theory obtained by compactifying the $6 \mathrm{~d}(2,0) A_{1}$ theory on a punctured Riemann surface $\mathcal{C}$. It is known that punctures on $\mathcal{C}$ can be "regular" or "irregular" depending on whether a six-dimensional BPS scalar operator has a simple pole or higher order pole at it. When the BPS operator has a pole of order $(n+1)$ at an irregular puncture, we say the puncture is of rank $n$. While this rank can be an integer or half-integer in general, we only consider integer ranks in this paper.

Let us first focus on the case in which $\mathcal{C}$ has no irregular puncture. In this case, the AGT correspondence [19] implies that the Nekrasov partition function of $\mathcal{T}_{\mathcal{C}}$ is identical to the conformal block of Liouville theory on $\mathcal{C}$, where the Liouville charge $Q$ is related to the $\Omega$-background parameters by $Q=\left(\epsilon_{1}+\epsilon_{2}\right) / \sqrt{\epsilon_{1} \epsilon_{2}}$. As an example, let us consider the 


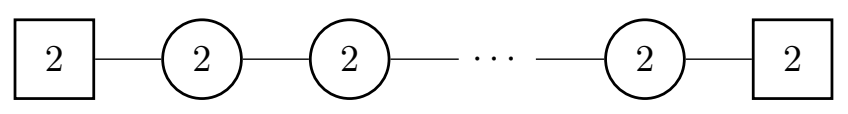

Figure 3. The quiver diagram of the class $\mathcal{S}$ theory $\mathcal{T}_{\mathcal{C}}$ for $\mathcal{C}$ being sphere with $(n+2)$ regular punctures. There are $(n-1)$ circles, each of which stands for an $\mathrm{SU}(2)$ gauge group.

case of $\mathcal{C}$ being a sphere with $(n+2)$ regular punctures. The corresponding $\mathcal{T}_{\mathcal{C}}$ is a linear quiver $\mathrm{SU}(2)$ gauge theory as shown in figure 3. The AGT correspondence implies

$$
\mathcal{Z}_{\mathrm{SU}(2)}\left(\vec{a} ; m_{1}, \cdots, m_{n+2}\right)=\mathcal{F}_{\alpha_{0}}{ }^{\alpha_{1}}{ }_{\beta_{1}}{ }^{\alpha_{2}}{ }_{\beta_{2}}{ }^{\alpha_{3}} \cdots_{\beta_{n-1}}{ }^{\alpha_{n}}{ }_{\alpha_{n+1}},
$$

where the 1.h.s. is the Nekrasov partition function of $\mathcal{T}_{C}$ with $\vec{a} \equiv\left(a_{1}, \cdots, a_{n-1}\right)$ being the VEVs of Coulomb branch operators, and $m_{i}$ being the masses of fundamental and bi-fundamental hypermultiplets. The subscript, $\mathrm{SU}(2)$, is for emphasizing that the gauge group of $\mathcal{T}_{C}$ is $\mathrm{SU}(2)^{n-1}$. The r.h.s. of (2.1) is the $(n+2)$-point conformal block of Liouville theory with $\beta_{i}$ being intermediate momenta, and $\alpha_{i}$ being external momenta. The $4 \mathrm{~d}$ and $2 \mathrm{~d}$ parameters are related by

$$
\frac{a_{i}}{\sqrt{\epsilon_{1} \epsilon_{2}}}=\beta_{i}+\frac{Q}{2}, \quad \frac{m_{i}}{\sqrt{\epsilon_{1} \epsilon_{2}}}=\alpha_{i}+\frac{Q}{2} .
$$

In the rest of this paper, we rescale dimensionful parameters so that $\epsilon_{1} \epsilon_{2}=1$, as in [19], and write things in terms of the $2 \mathrm{~d}$ parameters. When we need to recover the full $\epsilon_{i}$-dependence, we rescale the $2 \mathrm{~d}$ parameters as

$$
\alpha_{i} \rightarrow \frac{\alpha_{i}}{\sqrt{\epsilon_{1} \epsilon_{2}}}, \quad \beta_{i} \rightarrow \frac{\beta_{i}}{\sqrt{\epsilon_{1} \epsilon_{2}}}, \quad Q \rightarrow \frac{Q}{\sqrt{\epsilon_{1} \epsilon_{2}}} .
$$

The AGT correspondence has been generalized to the case in which $\mathcal{C}$ has irregular punctures $[17,18,20]$. The most important difference from the original AGT is that $\mathcal{T}_{\mathcal{C}}$ generically involves an $\mathrm{AD}$ theory [17, 18]. One simple example is the case in which $\mathcal{C}$ is a sphere with one irregular puncture of rank $n$ and one regular puncture. In this case, $\mathcal{T}_{\mathcal{C}}$ is an $\mathrm{AD}$ theory called the $\left(A_{1}, D_{2 n}\right)$ theory, and its partition function is identified as the two-point function of $2 \mathrm{~d}$ operators corresponding to the punctures on $\mathcal{C}$. By the state-operator map, this two-point function is mapped to the inner product of two states

$$
\mathcal{Z}_{\left(A_{1}, D_{2 n}\right)}=\left\langle a \mid I^{(n)}\right\rangle
$$

where $|a\rangle$ is the Virasoro primary state corresponding to the regular puncture, while $\left|I^{(n)}\right\rangle$ is a state corresponding to the rank- $n$ irregular puncture.

A more interesting situation arises when $\mathcal{C}$ has two irregular punctures of rank $n$. In this case, $\mathcal{T}_{\mathcal{C}}$ is no longer conformal, but is an $\mathrm{SU}(2)$ gauge theory coupled to two copies of $\left(A_{1}, D_{2 n}\right)$ theories. The quiver diagram of this gauge theory is shown in figure 2. Here, each $\left(A_{1}, D_{2 n}\right)$ in the matter sector is associated with an irregular puncture on $\mathcal{C}$. The $\beta$ function coefficient of this $\mathrm{SU}(2)$ gauge coupling is evaluated as $\beta_{0}=2 / n$, and therefore $\mathcal{T}_{\mathcal{C}}$ is never conformal. The generalized AGT correspondence then implies that the partition function of this theory is given by

$$
\mathcal{Z}_{\mathrm{SU}(2)}^{2 \times\left(A_{1}, D_{2 n}\right)}=\left\langle I^{(n)} \mid I^{(n)}\right\rangle,
$$

where $\left\langle I^{(n)}\right|$ and $\left|I^{(n)}\right\rangle$ correspond to the two irregular punctures on $\mathcal{C}$. 
In contrast to the state corresponding to a regular puncture, the state $\left|I^{(n)}\right\rangle$ is not a Virasoro primary but a linear combination of a primary and its descendants. This linear combination is determined to be a simultaneous solution to a set of equations. There are two characterizations of this set of equations, and here we follow the characterization proposed in $[18]:^{3}$

$$
L_{k}\left|I^{(n)}\right\rangle=\left\{\begin{array}{ll}
\lambda_{k}\left|I^{(n)}\right\rangle & \text { for } \quad n \leq k \leq 2 n \\
\left(\lambda_{k}+\sum_{\ell=1}^{n-k} \ell c_{\ell+k} \frac{\partial}{\partial c_{\ell}}\right)\left|I^{(n)}\right\rangle & \text { for } \quad 0 \leq k<n
\end{array},\right.
$$

where $\lambda_{k}$ are constants fixed by $c_{0}, \cdots, c_{n}$ as

$$
\lambda_{k}= \begin{cases}-\sum_{\ell=k-n}^{n} c_{\ell} c_{k-\ell} & \text { for } \quad n<k \leq 2 n \\ -\sum_{\ell=0}^{k} c_{\ell} c_{k-\ell}+(k+1) Q c_{k} & \text { for } \quad k \leq n\end{cases}
$$

It was conjectured in [18] that $\left|I^{(n)}\right\rangle$ satisfying the above equations exists in a highest weight module of Virasoro algebra. ${ }^{4}$

Since the constraints (2.6) are differential equations, $\left|I^{(n)}\right\rangle$ is not completely fixed by $c_{k}$ but depends on the "boundary condition" or the "asymptotic behavior." It was conjectured in [18] that $\left|I^{(n)}\right\rangle$ is uniquely fixed by specifying $n$ extra complex parameters in addition to $c_{0}, \cdots, c_{n-1}$ and $c_{n}$. These extra parameters characterize the asymptotic behavior of $\left|I^{(n)}\right\rangle$ in the small $c_{k}$ limit for $k=1, \cdots, n$. We denote these extra parameters by $\beta_{0}, \cdots, \beta_{n-1} .^{5}$ Then the generalized AGT correspondence implies that $c_{1}, \cdots, c_{n-1}$ are related to relevant couplings, $\beta_{1}, \cdots, \beta_{n-1}$ are related to the VEVs of Coulomb branch operators, and $c_{0}$ and $\beta_{0}$ are related to mass parameters of $\left(A_{1}, D_{2 n}\right)$ theory. The Liouville charge $Q$ is identified with $\epsilon_{1}+\epsilon_{2}$.

The irregular puncture of integer rank $n$ can be created by colliding $(n+1)$ regular punctures. As a result, the condition (2.6) is obtained in the colliding limit of Virasoro primary operators [18], which we briefly review here for later use. Let us first consider the state

$$
\left|\phi_{n}\left(z_{1}, \ldots, z_{n}\right)\right\rangle \equiv:\left(\prod_{i=1}^{n} V_{\alpha_{i}}^{L}\left(z_{i}\right)\right) V_{\alpha_{0}}^{L}(0):|0\rangle,
$$

where $V_{\alpha}^{L}(z)$ is the Virasoro primary vertex operator of conformal weight $\alpha(Q-\alpha)$, and $: X Y$ : is the normal-ordered product of $X$ and $Y$. The action of $T_{>}(y) \equiv \sum_{k \geq-1} y^{-k-2} L_{k}$ on this state is expressed as

$$
T_{>}(y)\left|\phi_{n}\left(z_{1}, \ldots, z_{n}\right)\right\rangle=\sum_{i=0}^{n}\left(\frac{\alpha_{i}\left(Q-\alpha_{i}\right)}{y-z_{i}}+\frac{1}{y-z_{i}} \frac{\partial}{\partial z_{i}}\right)\left|\phi_{n}\left(z_{1}, \ldots, z_{n}\right)\right\rangle,
$$

\footnotetext{
${ }^{3}$ The relation between the characterizations in [6] and [18] was partially studied in appendix B of [24].

${ }^{4}$ Note that the highest weight of this module is not necessarily equal to $c_{0}$. Indeed, the highest weight is given by $\beta_{0}$ discussed below.

${ }^{5}$ See section 3 and appendix B of [18] for more detail. Some explicit examples are also shown in [30].
} 
where $z_{0} \equiv 0$. The idea is then to consider a limit $z_{i} \rightarrow 0$ in which the above action of $T_{>}(y)$ remains well-defined but gives an interesting result. If we keep $\alpha_{i}$ finite in the limit, $\left|\phi_{n}\left(z_{1}, \cdots, z_{n}\right)\right\rangle$ just reduces to a single primary vertex operator acting on the vacuum. A more interesting limit is to take $z_{i} \rightarrow 0$ and $\alpha_{i} \rightarrow \infty$ with

$$
c_{k} \equiv \sum_{i=0}^{n} \alpha_{i} z_{i}^{k} \quad(k=0, \cdots, n),
$$

kept finite. We call this latter limit "colliding limit." It is straightforward to show that $T_{>}(y)$ acts on the state

$$
\left|I^{(n)}\right\rangle \equiv \lim _{\text {colliding limit }}\left|\phi_{n}\left(z_{1}, \cdots, z_{n}\right)\right\rangle,
$$

as

$$
T_{>}(y)\left|I^{(n)}\right\rangle=\left(\sum_{k=n}^{2 n} \frac{\lambda_{k}}{y^{k+2}}+\sum_{k=0}^{n-1} \frac{\lambda_{k}+\sum_{\ell=1}^{n-k} \ell c_{\ell+k} \frac{\partial}{\partial c_{\ell}}}{y^{k+2}}+\frac{L_{-1}}{y}\right)\left|I^{(n)}\right\rangle,
$$

where $\lambda_{i}$ are defined by (2.7). This implies that the state (2.11) satisfies (2.6), and therefore is an irregular state of rank $n$. Note that, since (2.8) is defined in terms of the normalordered product, (2.11) gives a well-defined state.

As mentioned already, the irregular state $\left|I^{(n)}\right\rangle$ generally depends on $n$ extra parameters, $\beta_{0}, \cdots, \beta_{n-1}$, corresponding to the "boundary condition" of a solution to the differential equations (2.6). These extra parameters correspond to inserting screening operators in the product (2.8). Since screening operators commute with the Virasoro algebra, this insertion does not break the conditions (2.6).

\section{Irregular states in $\operatorname{Vir} \oplus \boldsymbol{H}$ modules}

As reviewed in the previous section, the generalized AGT correspondence allows us to evaluate the partition function of $\mathrm{SU}(2)$ gauge theory coupled to two $\left(A_{1}, D_{2 n}\right)$ theories as in (2.5). In this section, we consider an extension of this generalized AGT correspondence to the case in which the gauge group is $\mathrm{U}(2)$ instead of $\mathrm{SU}(2)$. To that end, we start with the $\mathrm{U}(2)$ version of the original AGT correspondence and consider its colliding limit.

\subsection{U(2) version of the original AGT}

The U(2) version of the original AGT correspondence was studied in the literature. First, it was found in [19] that the Nekrasov partition function of a $U(2)$ gauge theory $\mathcal{Z}_{U(2)}$ is generally related to that of the $\mathrm{SU}(2)$ gauge theory with the same matter content, $\mathcal{Z}_{\mathrm{SU}(2)}$, by

$$
\mathcal{Z}_{\mathrm{U}(2)}=\mathcal{Z}_{\mathrm{SU}(2)} \mathcal{Z}_{\mathrm{U}(1)}
$$

where $\mathcal{Z}_{\mathrm{U}(1)}$ is called "U(1) factor" and regarded as the partition function of the $\mathrm{U}(1)$ part of the gauge theory. The AGT correspondence implies that $\mathcal{Z}_{\mathrm{SU}(2)}$ is identical to a conformal block of the 2d Liouville CFT. 
The $2 \mathrm{~d}$ interpretation of the $\mathrm{U}(1)$ factor was then given in [34]; $\mathcal{Z}_{\mathrm{U}(1)}$ is identical to a correlation function of chiral vertex operators for an extra Heisenberg algebra. To be concrete, let us focus on the linear quiver gauge theory described by the quiver in figure 3 . As shown in eq. (2.1), $\mathcal{Z}_{\mathrm{SU}(2)}$ is identified with the $(n+2)$-point conformal block of Liouville theory. On the other hand, the $\mathrm{U}(1)$ factor is identified as

$$
\mathcal{Z}_{\mathrm{U}(1)}=\left\langle V_{\alpha_{0}}^{H}\left(z_{0}\right) \cdots V_{\alpha_{n+1}}^{H}\left(z_{n+1}\right)\right\rangle
$$

where $V_{\alpha}^{H}(z) \equiv \exp \left(2(\alpha-Q) i \sum_{k<0} \frac{a_{k}}{k} z^{-k}\right) \exp \left(2 \alpha i \sum_{k>0} \frac{a_{k}}{k} z^{-k}\right)$, and the loci $z_{k}$ of the vertex operators coincide with those of the Liouville vertex operators in eq. (2.1). Here our convention for the Heisenberg algebra is such that $\left[a_{k}, a_{\ell}\right]=\frac{k}{2} \delta_{k+\ell, 0}$.

Combining (2.1), (3.1) and (3.2), we see that $\mathcal{Z}_{\mathrm{U}(2)}$ is identified with the correlator of $(n+2)$ chiral vertex operators of the form ${ }^{6}$

$$
\widehat{V}_{\alpha}(z) \equiv V_{\alpha}^{H}(z) \otimes V_{\alpha}^{L}(z),
$$

where $V_{\alpha}^{L}(z)$ is the Virasoro primary vertex operator of conformal weight $\alpha(Q-\alpha)$ in the Liouville CFT. Thus, the U(2) version of the AGT correspondence involves the direct sum of Virasoro and Heisenberg algebras, which we denote by $\operatorname{Vir} \oplus H$. Note that $L_{k}$ and $a_{k}$ are commutative since we consider the direct sum of the two algebras. The action of $\operatorname{Vir} \oplus H$ on $\widehat{V}_{\alpha}(z)$ is characterized by

$$
\begin{aligned}
& {\left[L_{n}, \widehat{V}_{\alpha}(z)\right]=\left(z^{n+1}+(n+1) \alpha(Q-\alpha) z^{n}\right) \widehat{V}_{\alpha}(z),} \\
& {\left[a_{n}, \widehat{V}_{\alpha}(z)\right]= \begin{cases}-i \alpha z^{n} \widehat{V}_{\alpha}(z) & (n<0) \\
i(Q-\alpha) z^{n} \widehat{V}_{\alpha}(z) & (n>0)\end{cases} }
\end{aligned}
$$

\section{2 $\mathrm{U}(2)$ version of the generalized AGT}

We now consider the $\mathrm{U}(2)$ version of the generalized AGT correspondence. Our idea is to start with the $\mathrm{U}(2)$ version of the original AGT, and take the same colliding limit as the one reviewed in the latter half of section 2 .

To that end, we start with the quiver gauge theory described in figure 3 with $\mathrm{U}(2)$ gauge groups. The partition function (3.1) is then identified with the product of (2.1) and (3.2). Note that the loci of the Heisenberg vertex operators in (3.2) coincide with those of the Liouville vertex operators, which reflects the fact that the $4 \mathrm{~d}$ gauge couplings of $\mathrm{U}(1) \subset \mathrm{U}(2)$ and $\mathrm{SU}(2) \subset \mathrm{U}(2)$ are identical. We now take the limit of parameters in which the $4 \mathrm{~d}$ theory flows to the theory described by the quiver in figure 2 with $\mathrm{U}(2)$ gauge group. On the $2 \mathrm{~d}$ side, this corresponds to the colliding limit of vertex operators (3.3), and gives rise to an irregular state $\left|\widehat{I}^{(n)}\right\rangle$ of $\operatorname{Vir} \oplus H$. The precise definition of $\left|\widehat{I}^{(n)}\right\rangle$ will be given below. The same argument as in section 2 then leads us to identifying the inner product

$$
\mathcal{Z}_{\mathrm{U}(2)}^{2 \times\left(A_{1}, D_{2 n}\right)}=\left\langle\widehat{I}^{(n)} \mid \widehat{I}^{(n)}\right\rangle
$$

as the partition function of $\mathrm{U}(2)$ gauge theory coupled to two $\left(A_{1}, D_{2 n}\right)$ theories.

\footnotetext{
${ }^{6}$ To be precise, one can also insert screening operators in the Liouville sector here.
} 
Our new irregular state $\left|\widehat{I}^{(n)}\right\rangle$ is characterized by the actions of the Virasoro and Heisenberg algebras on it. These actions can be read off by keeping track of their actions on $\widehat{V}_{\alpha_{k}}(z)=V_{\alpha_{k}}^{H}\left(z_{k}\right) \otimes V_{\alpha_{k}}^{L}\left(z_{k}\right)$ in the colliding limit. To see this, let us consider the state

$$
\left|\widehat{\phi}_{n}\left(z_{1}, \cdots, z_{n}\right)\right\rangle \equiv:\left(\prod_{i=1}^{n} \widehat{V}_{\alpha_{i}}\left(z_{i}\right)\right) \widehat{V}_{\alpha_{0}}(0):|0\rangle .
$$

The action of $T_{>}(y)$ on this state is the same as in eq. (2.9). Similarly, the action of $J_{>}(y) \equiv \sum_{k \geq 1} y^{-k-1} a_{k}$ is written as

$$
J_{>}(y)\left|\widehat{\phi}_{n}\left(z_{1}, \cdots, z_{n}\right)\right\rangle=-\sum_{i=0}^{n} \frac{i\left(Q-\alpha_{i}\right) z_{i}}{y\left(y-z_{i}\right)}\left|\widehat{\phi}\left(z_{1}, \cdots, z_{n}\right)\right\rangle,
$$

where $z_{0} \equiv 0$. We now take the colliding limit $z_{i} \rightarrow 0$ and $\alpha_{i} \rightarrow \infty$ with (2.10) kept fixed. The irregular state $\left|\widehat{I}^{(n)}\right\rangle$ is now defined by

$$
\left|\widehat{I}^{(n)}\right\rangle \equiv \lim _{\text {colliding limit }}\left|\widehat{\phi}_{n}\left(z_{1}, \cdots, z_{n}\right)\right\rangle .
$$

It is straightforward to show that $T_{>}(y)$ and $J_{>}(y)$ act on this state as

$$
\begin{aligned}
T_{>}(y)\left|\widehat{I}^{(n)}\right\rangle & =\left(\sum_{k=n}^{2 n} \frac{\lambda_{k}}{y^{k+2}}+\sum_{k=0}^{n-1} \frac{\lambda_{k}+\sum_{\ell=1}^{n-k} \ell c_{\ell+k} \frac{\partial}{\partial c_{\ell}}}{y^{k+2}}+\frac{L_{-1}}{y}\right), \\
J_{>}(y)\left|\widehat{I}^{(n)}\right\rangle & =\sum_{k=1}^{n} \frac{-i c_{k}}{y^{k+1}}\left|\widehat{I}^{(n)}\right\rangle
\end{aligned}
$$

where $\lambda_{k}$ and $c_{k}$ are given by (2.7) and (2.10), respectively. From the above result, we see that $\operatorname{Vir} \oplus H$ acts on $\left|\widehat{I}^{(n)}\right\rangle$ as

$$
\begin{aligned}
& L_{k}\left|\widehat{I}^{(n)}\right\rangle=\left\{\begin{array}{ll}
\lambda_{k}\left|\widehat{I}^{(n)}\right\rangle & \text { for } \quad n \leq k \leq 2 n \\
\left(\lambda_{k}+\sum_{\ell=1}^{n-k} \ell c_{\ell+k} \frac{\partial}{\partial c_{\ell}}\right)\left|\widehat{I}^{(n)}\right\rangle & \text { for } 0 \leq k<n
\end{array},\right. \\
& a_{k}\left|\widehat{I}^{(n)}\right\rangle=\left\{\begin{array}{ll}
0 & \text { for } \quad n<k \\
-i c_{k}\left|\widehat{I}^{(n)}\right\rangle & \text { for } 1 \leq k \leq n
\end{array} .\right.
\end{aligned}
$$

Note here that the above characterization of the irregular state does not fix the overall normalization, as in the case of the SU(2)-version of the generalized AGT correspondence. This means an ambiguity in the computation of the perturbative part of the partition function (3.6). However, it turns out that the instanton part of the partition function can be unambiguously computed, as will be discussed in the following sections.

Note also that $\left|\widehat{I}^{(n)}\right\rangle$ is by definition decomposed into the Virasoro part and the Heisenberg part as $\left|\widehat{I}^{(n)}\right\rangle=\left|I^{(n)}\right\rangle \otimes\left|I_{H}^{(n)}\right\rangle$, where $\left|I^{(n)}\right\rangle$ is the irregular state of Virasoro algebra that was reviewed in section 2. While $\left|I^{(n)}\right\rangle$ depends on $n$ extra parameters in addition 
to $c_{0}, \cdots, c_{n-1}$ and $c_{n}$, the state $\left|I_{H}^{(n)}\right\rangle$ is uniquely fixed by $c_{1}, \cdots, c_{n-1}$ and $c_{n}$ up to a prefactor. We here write down its explicit expression:

$$
\left|I_{H}^{(n)}\right\rangle=\exp \left(-2 i \sum_{k=1}^{n} \frac{c_{k}}{k} a_{-k}\right)|0\rangle,
$$

where the prefactor is fixed so that $\left|I_{H}^{(n)}\right\rangle$ reduces to $|0\rangle$ when $c_{k}=0$. Since $\left|I_{H}^{(n)}\right\rangle$ and $\left|I^{(n)}\right\rangle$ are respectively in a highest weight module of $V$ ir and $H$, we see that $\left|\widehat{I}^{(n)}\right\rangle$ is a state in a highest weight module of $\operatorname{Vir} \oplus H$. Note that the Heisenberg sector has no possible insertion of screening operators, and therefore (3.14) is the unique expression for $\left|I_{H}^{(n)}\right\rangle$ up to a prefactor. Indeed, the constraints (3.13) are eigenstate equations, whose solution is fixed (up to the normalization) by $\left\{c_{k}\right\}$ without specifying a "boundary condition."

Given the U(2)-version of the generalized AGT correspondence (3.6), one can now study the decomposition of $\mathcal{Z}_{\mathrm{U}(2)}^{2 \times\left(A_{1}, D_{2 n}\right)}$ as a sum over pairs of Young diagrams as in eq. (1.2). In the next section, we explicitly evaluate this decomposition to read off the factor $\mathcal{Z}_{Y_{1}, Y_{2}}^{\left(A_{1}, D_{2 n}\right)}$ in $(1.2)$.

\section{Nekrasov-type formula for AD matter}

Here we read off the factor $\mathcal{Z}_{Y_{1}, Y_{2}}^{\left(A_{1}, D_{2 n}\right)}$ in (1.2) from the $\mathrm{U}(2)$-version of the generalized AGT correspondence (3.6). This factor can be interpreted as the contribution of the $\left(A_{1}, D_{2 n}\right)$ theory at the fixed point corresponding to $\left(Y_{1}, Y_{2}\right)$ on the $\mathrm{U}(2)$ instanton moduli space.

\subsection{Decomposition}

To read off $\mathcal{Z}_{Y_{1}, Y_{2}}^{\left(A_{1}, D_{2 n}\right)}$, we first decompose (3.6) as a sum over pairs of Young diagrams, using a nice basis of highest weight modules of $\operatorname{Vir} \oplus H$ that was found in [34]. To describe it, let us denote by

$$
\mathcal{Z}_{Y_{1}, Y_{2} ; W_{1}, W_{2}}^{\text {bifund }}(a, b, \alpha)
$$

the contribution to the Nekrasov partition function from a bi-fundamental hypermultiplet of $\mathrm{U}(2) \times \mathrm{U}(2)$. Here, $(a, b)$ stands for the VEVs of Coulomb branch operators in the vector multiplet for $\mathrm{SU}(2) \times \mathrm{SU}(2) \subset \mathrm{U}(2) \times \mathrm{U}(2)$, and $\alpha$ is a mass parameter. The explicit expression for (4.1) is written in appendix A. It was shown in [34] that there exists an orthogonal basis, $\left|a ; Y_{1}, Y_{2}\right\rangle$, of the highest weight module of $\operatorname{Vir} \oplus H$ such that

$$
\frac{\left\langle a ; Y_{1}, Y_{2}\left|V_{\alpha}(1)\right| b ; W_{1}, W_{2}\right\rangle}{\left\langle a\left|V_{\alpha}(1)\right| b\right\rangle}=\mathcal{Z}_{Y_{1}, Y_{2} ; W_{1}, W_{2}}^{\text {bifund }}(a, b, \alpha),
$$

where $|a\rangle$ is the highest weight state satisfying $L_{0}|a\rangle=\Delta(a)|a\rangle$ and $L_{n}|a\rangle=a_{n}|a\rangle=0$ for $n>0, V_{\alpha}(z)$ is the vertex operator shown in (3.3), and $Y_{k}$ and $W_{k}$ are arbitrary Young diagrams. Note that the conjugate $\left\langle a ; Y_{1}, Y_{2}\right|$ is not the usual hermitian conjugate of $\left|a ; Y_{1}, Y_{2}\right\rangle$; it is obtained by expanding $\left|a ; Y_{1}, Y_{2}\right\rangle$ as a linear combination of $L_{-k_{1}}^{m_{1}} \cdots L_{-k_{p}}^{m_{p}} a_{-\ell_{1}}^{n_{1}} \cdots a_{-\ell_{q}}^{n_{q}}|a\rangle$, and then replacing each such state with $\langle a| L_{k_{p}}^{m_{p}} \cdots L_{k_{1}}^{m_{1}} a_{\ell_{q}}^{n_{q}} \cdots a_{\ell_{1}}^{n_{1}}$ without changing the coefficients. The first few examples of $\left|a ; Y_{1}, Y_{2}\right\rangle$ are presented in appendix B. It was also 
shown in [34] that $\left|a ; Y_{1}, Y_{2}\right\rangle$ is generally a linear combination of descendants of $|a\rangle$ at level $\left(\left|Y_{1}\right|+\left|Y_{2}\right|\right) .^{7}$

As discussed in [34], the condition (4.2) and the fact that $\mathcal{Z}_{Y_{1}, Y_{2}}^{\mathrm{vec}}(a)=1 / \mathcal{Z}_{Y_{1}, Y_{2} ; Y_{1}, Y_{2}}^{\text {bifund }}(a, a, 0)$ imply

$$
\mathbf{1}=\sum_{Y_{1}, Y_{2}} \mathcal{Z}_{Y_{1}, Y_{2}}^{\mathrm{vec}}(a)\left|a ; Y_{1}, Y_{2}\right\rangle\left\langle a ; Y_{1}, Y_{2}\right|
$$

on the highest weight $\operatorname{Vir} \oplus H$-module associated with $|a\rangle$. Note again that $\left\langle a ; Y_{1}, Y_{2}\right|$ is not the usual conjugate of $\left|a ; Y_{1}, Y_{2}\right\rangle$. Let us now take $|a\rangle$ to be the highest weight state of the $\operatorname{Vir} \oplus H$-module that includes $\left|\widehat{I}^{(n)}\right\rangle$. Then, by inserting (4.3) in eq. (3.6), one obtains the following decomposition:

$$
\mathcal{Z}_{\mathrm{U}(2)}^{2 \times\left(A_{1}, D_{2 n}\right)}=\sum_{Y_{1}, Y_{2}} \mathcal{Z}_{Y_{1}, Y_{2}}^{\mathrm{vec}}(a)\left\langle\widehat{I}^{(n)} \mid a ; Y_{1}, Y_{2}\right\rangle\left\langle a ; Y_{1}, Y_{2} \mid \widehat{I}^{(n)}\right\rangle
$$

We interpret the above expression as a sum over fixed points on the moduli space of $\mathrm{U}(2)$ instantons, and $\left\langle a ; Y_{1}, Y_{2} \mid \widehat{I}^{(n)}\right\rangle$ and $\left\langle\widehat{I}^{(n)} \mid a ; Y_{1}, Y_{2}\right\rangle$ as the contributions of the $\left(A_{1}, D_{2 n}\right)$ theories corresponding to $\left|\widehat{I}^{(n)}\right\rangle$ and $\left\langle\widehat{I}^{(n)}\right|$, respectively. Note that this particularly implies that $\left\langle a ; \emptyset, \emptyset \mid \widehat{I}^{(n)}\right\rangle$ and $\left\langle\widehat{I}^{(n)} \mid a ; \emptyset, \emptyset\right\rangle$ are the partition function of $\left(A_{1}, D_{2 n}\right)$ theory with its flavor symmetry un-gauged. Since $|a ; \emptyset, \emptyset\rangle=|a\rangle[34]$, this is indeed consistent with (2.4).

\subsection{Identification of $\mathcal{Z}_{Y_{1}, Y_{2}}^{\left(A_{1}, D_{2 n}\right)}$}

Comparing (4.4) with (1.2), we see that it is natural to interpret

$$
\mathcal{Z}_{Y_{1}, Y_{2}}^{\left(A_{1}, D_{2 n}\right)} \sim \frac{\left\langle a ; Y_{1}, Y_{2} \mid \widehat{I}^{(n)}\right\rangle}{\left\langle a \mid \widehat{I}^{(n)}\right\rangle}, \quad \widetilde{\mathcal{Z}}_{Y_{1}, Y_{2}}^{\left(A_{1}, D_{2 n}\right)} \sim \frac{\left\langle\widehat{I}^{(n)} \mid a ; Y_{1}, Y_{2}\right\rangle}{\left\langle\widehat{I}^{(n)} \mid a\right\rangle},
$$

with possible proportionality constants. Note that the denominators in (4.5) are necessary for $\mathcal{Z}_{\emptyset, \emptyset}^{\left(A_{1}, D_{2 n}\right)}=\widetilde{\mathcal{Z}}_{\emptyset, \emptyset}^{\left(A_{1}, D_{2 n}\right)}=1$.

Here, $\left|\widehat{I}^{(n)}\right\rangle$ and $\left\langle\widehat{I}^{(n)}\right|$ correspond to two different $\left(A_{1}, D_{2 n}\right)$ theories. Indeed, as seen from their colliding-limit derivation, these $\left(A_{1}, D_{2 n}\right)$ theories are differently coupled to the $\mathrm{U}(1)$-part of the gauge group. Therefore, $\mathcal{Z}_{Y_{1}, Y_{2}}^{\left(A_{1}, D_{2 n}\right)}$ and $\widetilde{Z}_{Y_{1}, Y_{2}}^{\left(A_{1}, D_{2 n}\right)}$ are not identical.

In the rest of this sub-section, we make the relations (4.5) more precise. To that end, we first focus on the left relation, and read off how the $4 \mathrm{~d}$ and $2 \mathrm{~d}$ parameters are related. Note that $\left\langle a ; Y_{1}, Y_{2} \mid \widehat{I}^{(n)}\right\rangle$ on the r.h.s. depends on $(2 n+1)$ parameters. Indeed, $\left|\widehat{I}^{(n)}\right\rangle$ depends on $n$ extra parameters $\beta_{0}, \cdots, \beta_{n-1}$ in addition to $c_{0}, \cdots, c_{n}$, as reviewed in section 3.2. One of these extra parameters fixes the highest weight, $a$, of the $V i r \oplus H$-module that includes $\left|\widehat{I}^{(n)}\right\rangle$, and therefore $\left\langle a ; Y_{1}, Y_{2} \mid \widehat{I}^{(n)}\right\rangle$ is completely fixed by these $(2 n+1)$ parameters. On the other hand, $\mathcal{Z}_{Y_{1}, Y_{2}}^{\left(A_{1}, D_{2 n}\right)}(a, m, \boldsymbol{d}, \boldsymbol{u})$ on the l.h.s. depends only on $2 n$ parameters, $a, m, \boldsymbol{d}=\left(d_{1}, \cdots, d_{n-1}\right)$ and $\boldsymbol{u}=\left(u_{1}, \cdots, u_{n-1}\right)$. Here, $a$ and $\boldsymbol{u}$ are the VEVs of Coulomb branch operators, $\boldsymbol{d}$ are relevant couplings and $m$ is a mass parameter. Therefore, there is a discrepancy in the number of parameters between the $2 \mathrm{~d}$ and $4 \mathrm{~d}$ sides.

\footnotetext{
${ }^{7}$ Here, the "level" is defined by the sum of the levels of the Virasoro and Heisenberg parts. For example, the level of $L_{-k_{1}}\left(L_{-k_{2}}\right)^{3} a_{-\ell}|a\rangle$ is $k_{1}+3 k_{2}+\ell$.
} 
To see this discrepancy more explicitly, let us identify the precise relation between the $2 \mathrm{~d}$ and $4 \mathrm{~d}$ parameters. We start with the Seiberg-Witten (SW) curve of the $\left(A_{1}, D_{2 n}\right)$ theory $[17,31]$

$$
x^{2}=\frac{a^{2}}{z^{2}}+\sum_{k=1}^{n-1} \frac{u_{k}}{z^{n+2-k}}+\frac{m}{z^{n+2}}+\sum_{k=1}^{n-1} \frac{d_{k}}{z^{2 n+2-k}}+\frac{1}{z^{2 n+2}},
$$

where the SW 1-form is given by $x d z .{ }^{8}$ This curve is identified, on the $2 \mathrm{~d}$ side, as the classical limit $\epsilon_{i} \rightarrow 0$ of the following [19]

$$
x^{2}=-\frac{\left\langle a|T(z)| \widehat{I}^{(n)}\right\rangle}{\left\langle a \mid \widehat{I}^{(n)}\right\rangle}=-\frac{\Delta(a)}{z^{2}}+\cdots+\frac{2 c_{n} c_{n-1}}{z^{2 n+1}}+\frac{c_{n}^{2}}{z^{2 n+2}},
$$

up to a change of variables that preserves the SW 1-form. Note that the r.h.s. of the above equation can be explicitly evaluated via eq. (3.12). ${ }^{9}$ We here change the variables in (4.7) as $z \rightarrow\left(c_{n}\right)^{\frac{1}{n}} z$ and $x \rightarrow\left(c_{n}\right)^{-\frac{1}{n}} x$ so that the coefficient of $1 / z^{2 n+2}$ is 1 . Comparing the classical limit of the resulting equation with (4.6), we obtain the relation between the $2 \mathrm{~d}$ and $4 \mathrm{~d}$ parameters. ${ }^{10}$ To make this relation simple, let us define on the $2 \mathrm{~d}$ side

$$
\gamma_{k} \equiv \frac{c_{k}}{\left(c_{n}\right)^{\frac{k}{n}}}
$$

for $k=0, \cdots, n-1$. In terms of these variables, the relation between the $2 \mathrm{~d}$ and $4 \mathrm{~d}$ parameters is expressed as

$$
d_{k}=\sum_{\ell=n-k}^{n} \gamma_{\ell} \gamma_{2 n-k-\ell}, \quad m=\sum_{\ell=0}^{n} \gamma_{\ell} \gamma_{n-\ell}, \quad u_{k}=\sum_{\ell=0}^{n-k} \gamma_{\ell} \gamma_{n-k-\ell}-\sum_{\ell=1}^{k} \ell \gamma_{\ell+n-k} \frac{\partial}{\partial \gamma_{\ell}} \mathcal{F}_{\left(A_{1}, D_{2 n}\right)},
$$

where the derivatives $\partial / \partial \gamma_{\ell}$ are defined with $\vec{\gamma} \equiv\left(\gamma_{0}, \cdots, \gamma_{n-1}\right)$ and $c_{n}$ taken as independent variables, and $\mathcal{F}_{\left(A_{1}, D_{2 n}\right)}$ is the classical limit of $\log \left\langle a \mid \widehat{I}^{(n)}\right\rangle$. These expressions imply that, when one takes $\vec{\gamma}$ and $c_{n}$ as independent variables, all the $4 \mathrm{~d}$ parameters are independent of $c_{n} \cdot{ }^{11}$ This reflects the conformal invariance of $\left(A_{1}, D_{2 n}\right)$, and explains the discrepancy in the number of parameters between the $2 \mathrm{~d}$ and $4 \mathrm{~d}$ sides.

The above discussion implies that, for the left relation in (4.5) to be an equality, the $c_{n^{-}}$ dependence of the r.h.s. needs to be canceled by a constant of proportionality. To identify

\footnotetext{
${ }^{8}$ Here $a$ is regarded as the mass parameter corresponding to a flavor $\mathrm{SU}(2)$ sub-group of the $\left(A_{1}, D_{2 n}\right)$ theory.

${ }^{9}$ Recall here that, to recover the full $\epsilon_{i}$-dependence, one needs to perform a replacement corresponding to (2.3). For the irregular state $\left|I^{(n)}\right\rangle$, this replacement implies $c_{k} \rightarrow c_{k} / \sqrt{\epsilon_{1} \epsilon_{2}}$ as seen from its colliding-limit derivation.

${ }^{10}$ Note here that $\Delta(a)$ reduces to $-a^{2}$ in the classical limit $\epsilon_{1}, \epsilon_{2} \rightarrow 0$.

${ }^{11}$ To prove this statement, one needs to show that $\frac{\partial}{\partial \gamma_{\ell}} \mathcal{F}_{\left(A_{1}, D_{2 n}\right)}$ is independent of $c_{n}$. This can be shown as follows. As we will show below, $\left\langle a ; Y_{1}, Y_{2} \mid \widehat{I}^{(n)}\right\rangle=\left(c_{n}\right)^{\frac{\Delta_{a}-\Delta_{c_{0}}+\left|Y_{1}\right|+\left|Y_{2}\right|}{n}} f_{Y_{1}, Y_{2}}(\vec{\gamma})$ for a function $f_{Y_{1}, Y_{2}}(\vec{\gamma})$ independent of $c_{n}$. Setting $Y_{1}=Y_{2}=\emptyset$, we find $\log \left\langle a \mid \widehat{I}^{(n)}\right\rangle=\frac{\Delta_{a}-\Delta_{c_{0}}}{n} \log c_{n}+\log f_{\emptyset, \emptyset}(\vec{\gamma})$. This implies that $\frac{\partial}{\partial \gamma_{\ell}} \log \left\langle a \mid \widehat{I}^{(n)}\right\rangle=\frac{\partial}{\partial \gamma_{\ell}} \log f_{\emptyset, \emptyset}(\vec{\gamma})$. Since this is independent of $c_{n}$, its classical limit $\frac{\partial}{\partial \gamma_{\ell}} \mathcal{F}_{\left(A_{1}, D_{2 n}\right)}$ is also independent of $c_{n}$ when written in terms of $\vec{\gamma}$ and $c_{n}$.
} 
this proportionality constant, let us consider

$$
\left.n c_{n} \frac{\partial}{\partial c_{n}}\right|_{\vec{\gamma}}\left\langle a ; Y_{1}, Y_{2} \mid \widehat{I}^{(n)}\right\rangle
$$

where $\partial /\left.\partial c_{n}\right|_{\vec{\gamma}}$ is the derivative with respect to $c_{n}$ with $\vec{\gamma}=\left(\gamma_{0}, \cdots, \gamma_{n-1}\right)$ kept fixed. From (3.12), we see that this is identical to

$$
\left\langle a ; Y_{1}, Y_{2}\left|\left(L_{0}-\Delta_{c_{0}}\right)\right| \widehat{I}^{(n)}\right\rangle=\left(\Delta_{a}-\Delta_{c_{0}}+\left|Y_{1}\right|+\left|Y_{2}\right|\right)\left\langle a ; Y_{1}, Y_{2} \mid \widehat{I}^{(n)}\right\rangle .
$$

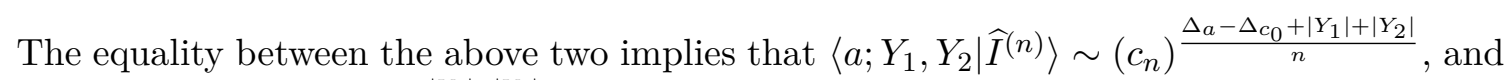
therefore the ratio $\left(c_{n}\right)^{-\frac{\left|Y_{1}\right|+\left|Y_{2}\right|}{n}}\left\langle a ; Y_{1}, Y_{2} \mid \widehat{I}^{(n)}\right\rangle /\left\langle a \mid \widehat{I}^{(n)}\right\rangle$ is independent of $c_{n}$ when written in terms of $\vec{\gamma}$ and $c_{n}{ }^{12}$ This suggests the following identification

$$
\mathcal{Z}_{Y_{1}, Y_{2}}^{\left(A_{1}, D_{2 n}\right)}=\left(\zeta c_{n}\right)^{-\frac{\left|Y_{1}\right|+\left|Y_{2}\right|}{n}} \frac{\left\langle a ; Y_{1}, Y_{2} \mid \widehat{I}^{(n)}\right\rangle}{\left\langle a \mid \widehat{I}^{(n)}\right\rangle},
$$

where $\zeta$ is a possible numerical constant independent of all variables. Note that $\zeta$ above can be absorbed by rescaling the instanton factor $\Lambda$.

A parallel discussion shows that the parameters of $\widetilde{\mathcal{Z}}_{Y_{1}, Y_{2}}(a, \widetilde{m}, \widetilde{\boldsymbol{d}}, \widetilde{\boldsymbol{u}})$ are related to those of $\left\langle\widehat{I}^{(n)} \mid a ; Y_{1}, Y_{2}\right\rangle$ by a similar relation to (4.9). As $\left\langle a ; Y_{1}, Y_{2} \mid \widehat{I}^{(n)}\right\rangle$ depends on $c_{0}, \cdots, c_{n}$ and $\beta_{0}, \cdots, \beta_{n-1},\left\langle\widehat{I}^{(n)} \mid a ; Y_{1}, Y_{2}\right\rangle$ also depends on $(2 n+1)$ parameters, which we denote by $\widetilde{c}_{0}, \cdots, \widetilde{c}_{n}$ and $\widetilde{\beta}_{0}, \cdots, \widetilde{\beta}_{n-1}$. From the same argument as above, we see that

$$
\widetilde{\mathcal{Z}}_{Y_{1}, Y_{2}}^{\left(A_{1}, D_{2 n}\right)}=\left(-\zeta \widetilde{c}_{n}^{*}\right)^{-\frac{\left|Y_{1}\right|+\left|Y_{2}\right|}{n}} \frac{\left\langle\widehat{I}^{(n)} \mid a ; Y_{1}, Y_{2}\right\rangle}{\left\langle\widehat{I}^{(n)} \mid a\right\rangle},
$$

where $\widetilde{c}_{n}^{*}$ is the complex conjugate of $\widetilde{c}_{n} \cdot{ }^{13}$

In the above identifications, $\mathcal{Z}_{Y_{1}, Y_{2}}^{\left(A_{1}, D_{2 n}\right)}$ and $\widetilde{\mathcal{Z}}_{Y_{1}, Y_{2}}^{\left(A_{1}, D_{2 n}\right)}$ are regarded as the contribution of the $\left(A_{1}, D_{2 n}\right)$ theory corresponding to $\left|\widehat{I}^{(n)}\right\rangle$ and $\left\langle\widehat{I}^{(n)}\right|$, respectively. As discussed at the beginning, these two $\left(A_{1}, D_{2 n}\right)$ theories have different couplings to the $\mathrm{U}(1)$ part of the gauge group. As we will see in section 4.4 , the difference between $\mathcal{Z}_{Y_{1}, Y_{2}}^{\left(A_{1}, D_{2 n}\right)}$ and $\widetilde{\mathcal{Z}}_{Y_{1}, Y_{2}}^{\left(A_{1}, D_{2 n}\right)}$ is an $\mathrm{AD}$ counterpart of the difference between the fundamental and anti-fundamental hypermultiplets of $\mathrm{U}(2)$.

\footnotetext{
${ }^{12}$ Recall here that $\langle a ; \emptyset, \emptyset|=\langle a|$.

${ }^{13}$ The opposite sign in the bracket in $\left(-\widetilde{c}_{n}^{*}\right)^{-\frac{\left|Y_{1}\right|+\left|Y_{2}\right|}{n}}$ can be understood as follows. First recall that $\left\langle a ; Y_{1}, Y_{2}\right|$ in (4.13) is not the usual conjugate of $\left|a ; Y_{1}, Y_{2}\right\rangle$, as discussed in [34]. Indeed, $\left\langle a ; Y_{1}, Y_{2}\right|$ is obtained by expanding $\left|a ; Y_{1}, Y_{2}\right\rangle$ as a linear combination of $L_{-k_{1}}^{m_{1}} \cdots L_{-k_{p}}^{m_{p}} a_{-\ell_{1}}^{n_{1}} \cdots a_{-\ell_{q}}^{n_{q}}|a\rangle$, and then replacing each such state with $\langle a| L_{k_{p}}^{m_{p}} \cdots L_{k_{1}}^{m_{1}} a_{\ell_{q}}^{n_{q}} \cdots a_{\ell_{1}}^{n_{1}}$ without changing the coefficients. This implies that, $\left\langle\widehat{I}^{(n)} \mid a ; Y_{1}, Y_{2}\right\rangle$ is obtained from $\left\langle a ; Y_{1}, Y_{2} \mid \widehat{I}^{(n)}\right\rangle$ by replacing $\left\langle a\left|L_{k_{p}}^{m_{p}} \cdots L_{k_{1}}^{m_{1}} a_{\ell_{q}}^{n_{q}} \cdots a_{\ell_{1}}^{n_{1}}\right| \widehat{I}^{(n)}\right\rangle$ with $\left\langle\widehat{I}^{(n)}\left|L_{-k_{p}}^{m_{p}} \cdots L_{-k_{1}}^{m_{1}} a_{-\ell_{q}}^{n_{q}} \cdots a_{-\ell_{1}}^{n_{1}}\right| a\right\rangle$. From (3.12) and (3.13), we see that this is equivalent to the replacement

$$
c_{k} \longrightarrow-\widetilde{c}_{k}^{*}, \quad Q \rightarrow-Q^{*}
$$

for $k=0, \cdots, n$. In particular, $c_{n}$ in (4.12) is replaced by $-\widetilde{c}_{n}^{*}$. 


\subsection{Identification of $\Lambda$}

The identifications (4.12) and (4.13) imply that (4.4) is re-expressed as

$\mathcal{Z}_{Y_{1}, Y_{2}}^{2 \times\left(A_{1}, D_{2 n}\right)}=\mathcal{Z}_{\text {pert }} \sum_{Y_{1}, Y_{2}}\left(-\zeta^{2} c_{n} \widetilde{c}_{n}^{*}\right)^{\frac{\left|Y_{1}\right|+\left|Y_{2}\right|}{n}} \mathcal{Z}_{Y_{1}, Y_{2}}^{\mathrm{vec}}(a) \mathcal{Z}_{Y_{1}, Y_{2}}^{\left(A_{1}, D_{2 n}\right)}(a, m, \boldsymbol{d}, \boldsymbol{u}) \widetilde{\mathcal{Z}}_{Y_{1}, Y_{2}}^{\left(A_{1}, D_{2 n}\right)}(a, \widetilde{m}, \widetilde{\boldsymbol{d}}, \widetilde{\boldsymbol{u}})$,

with the perturbative part $\mathcal{Z}_{\text {pert }} \equiv\left\langle\widehat{I}^{(n)} \mid a\right\rangle\left\langle a \mid \widehat{I}^{(n)}\right\rangle$. Comparing (4.15) with (1.2), we identify the $4 \mathrm{~d}$ dynamical scale as

$$
\Lambda^{2}=-\zeta^{2} c_{n} \widetilde{c}_{n}^{*}
$$

Recall that the $\left(A_{1}, D_{2 n}\right)$ sector is independent of $c_{n}$ (and $\left.\widetilde{c}_{n}^{*}\right)$ as a result of its conformal invariance. Here, the $\mathrm{U}(2)$ gauge coupling breaks this conformal invariance through the dynamical scale, and therefore it is natural that $\Lambda$ depends on $c_{n}$ and $\widetilde{c}_{n}^{*}$.

The identification (4.16) is also consistent with the SW curve. The curve of the theory shown in figure 2 is written as [17]

$$
x^{2}=\Lambda_{0}^{2} z^{2 n-2}+\cdots+\frac{\Lambda_{0}^{2}}{z^{2 n+2}},
$$

where $\Lambda_{0}$ is a dynamical scale that can differ from $\Lambda$ by a numerical factor, and the ellipsis stands for a Laurent polynomial of $z$ which is less singular than $z^{2 n-2}$ at $z=\infty$ and than $1 / z^{2 n+2}$ at $z=0 .{ }^{14}$ Now, by the same argument as around eq. (4.7), this curve is identified as

$$
x^{2}=-\frac{\left\langle\widehat{I}^{(n)}|T(z)| \widehat{I}^{(n)}\right\rangle}{\left\langle\widehat{I}^{(n)} \mid \widehat{I}^{(n)}\right\rangle}=\left(\widetilde{c}_{n}^{*}\right)^{2} z^{2 n-2}+\cdots+\frac{\left(c_{n}\right)^{2}}{z^{2 n+2}},
$$

up to a change of variables that preserves the SW 1-form. After changing variables as $z \rightarrow z\left(-c_{n} / \widetilde{c}_{n}^{*}\right)^{\frac{1}{2 n}}$ and $x \rightarrow x\left(-c_{n} / \widetilde{c}_{n}^{*}\right)^{-\frac{1}{2 n}}$, the curve (4.18) is re-expressed as $x^{2}=$ $\left(-c_{n} \widetilde{c}_{n}^{*}\right) z^{2 n-2}+\cdots+\left(-c_{n} \widetilde{c}_{n}^{*}\right) / z^{2 n+2}$. Comparing this with (4.17), we find $\Lambda_{0}^{2}=-c_{n} \widetilde{c}_{n}^{*}$, which coincides with (4.16) up to a numerical factor.

\subsection{Consistency check}

Since the $\left(A_{1}, D_{2}\right)$ theory is a theory of free hypermultiplets in the doublet of $\mathrm{U}(2)$, one can perform a consistency check of our proposals (4.12) and (4.13) by comparing them with the Nekrasov's formula for fundamental and anti-fundamental hypermultiplets.

Let us first consider (4.12). In the case of $n=1$, the irregular state involved in (4.12) satisfies

$$
\begin{aligned}
L_{2}\left|\widehat{I}^{(1)}\right\rangle & =-c_{1}^{2}\left|\widehat{I}^{(1)}\right\rangle, \\
L_{1}\left|\widehat{I}^{(1)}\right\rangle & =2\left(Q-c_{0}\right) c_{1}\left|\widehat{I}^{(1)}\right\rangle, \\
L_{0}\left|\widehat{I}^{(1)}\right\rangle & =\left(\Delta_{c_{0}}+c_{1} \frac{\partial}{\partial c_{1}}\right)\left|\widehat{I}^{(1)}\right\rangle, \\
a_{1}\left|\widehat{I}^{(1)}\right\rangle & =-i c_{1}\left|\widehat{I}^{(1)}\right\rangle,
\end{aligned}
$$

\footnotetext{
${ }^{14}$ The SW 1-form is again given by $x d z$.
} 
together with $a_{k}\left|\widehat{I}^{(1)}\right\rangle=0$ for $k \geq 2$. These equations are enough to compute the ratio of inner products $\left\langle a ; Y_{1}, Y_{2} \mid \widehat{I}^{(1)}\right\rangle /\left\langle a \mid \widehat{I}^{(1)}\right\rangle$. We then find that ${ }^{15}$

$$
\left(-\frac{c_{1}}{2}\right)^{-\left|Y_{1}\right|-\left|Y_{2}\right|} \frac{\left\langle a ; Y_{1}, Y_{2} \mid \widehat{I}^{(1)}\right\rangle}{\left\langle a \mid \widehat{I}^{(1)}\right\rangle}=\mathcal{Z}_{Y_{1}, Y_{2}}^{\text {fund }}(a, m),
$$

where $\mathcal{Z}_{Y_{1}, Y_{2}}^{\text {fund }}$ is the contribution from a fundamental hypermultiplet of $\mathrm{U}(2)$ as reviewed in appendix A. The mass parameter $m$ is related to $c_{0}$ by

$$
m=c_{0}-\frac{Q}{2},
$$

which coincides with (4.9) in the classical limit $\epsilon_{i} \rightarrow 0$. We see that (4.23) is perfectly consistent with our proposal (4.12) for $\zeta=-1 / 2$.

We also perform the same computation for $\left\langle\widehat{I}^{(1)} \mid a ; Y_{1}, Y_{2}\right\rangle /\left\langle\widehat{I}^{(1)} \mid a\right\rangle$ to find that ${ }^{16}$

$$
\left(\frac{\widetilde{c}_{n}^{*}}{2}\right)^{-\left|Y_{1}\right|-\left|Y_{2}\right|} \frac{\left\langle\widehat{I}^{(1)} \mid a ; Y_{1}, Y_{2}\right\rangle}{\left\langle\widehat{I}^{(1)} \mid a\right\rangle}=\mathcal{Z}_{Y_{1}, Y_{2}}^{\text {anti-fund }}(a, \widetilde{m}),
$$

where $\widetilde{m}$ is similarly identified as

$$
\widetilde{m}=\left(\widetilde{c}_{0}-\frac{Q}{2}\right)^{*}
$$

This is also in perfect agreement with our proposal (4.13) for $\zeta=-1 / 2$.

The above two checks suggest that the difference between $\mathcal{Z}_{Y_{1}, Y_{2}}^{\left(A_{1}, D_{2 n}\right)}$ and $\widetilde{\mathcal{Z}}_{Y_{1}, Y_{2}}^{\left(A_{1}, D_{2 n}\right)}$ can be regarded as the $\mathrm{AD}$ counterpart of the difference between the fundamental and anti-fundamental hypermultiplets.

\section{Application to $\left(A_{3}, A_{3}\right)$ theory}

In this section, we apply our method to the $\left(A_{3}, A_{3}\right)$ theory and compute its partition function. Recall that the $\left(A_{3}, A_{3}\right)$ theory is described by the quiver diagram in figure 1 . When the gauge group is replaced by $\mathrm{U}(2)$, the partition function of the theory is given by

$$
\mathcal{Z}_{\mathrm{U}(2)}=\mathcal{Z}_{\text {pert }}^{\mathrm{U}(2)} \sum_{Y_{1}, Y_{2}} q^{\left|Y_{1}\right|+\left|Y_{2}\right|} \mathcal{Z}_{Y_{1}, Y_{2}}^{\mathrm{vec}}(a) \mathcal{Z}_{Y_{1}, Y_{2}}^{\mathrm{fund}}(a, M) \prod_{i=1}^{2} \mathcal{Z}_{Y_{1}, Y_{2}}^{\left(A_{1}, D_{4}\right)}\left(a, m_{i}, d_{i}, u_{i}\right)
$$

where $\mathcal{Z}_{Y_{1}, Y_{2}}^{\left(A_{1}, D_{4}\right)}$ is the contribution of the $\left(A_{1}, D_{4}\right)$ theory that we have identified in eq. (4.12), and $\mathcal{Z}_{\text {pert }}^{\mathrm{U}(2)}$ is the perturbative contribution that makes the series in $q$ start with 1 . The parameters $m_{i}, d_{i}$ and $u_{i}$ are respectively a mass parameter, relevant coupling of dimension $1 / 2$, and the VEV of Coulomb branch operator of dimension $3 / 2$ in the $i$-th $\left(A_{1}, D_{4}\right)$ theory. Since the $\mathrm{SU}(2)$ gauge coupling is exactly marginal, the above expression includes the exponential of the marginal gauge coupling, $q$, instead of a dynamical scale.

\footnotetext{
${ }^{15}$ We checked this equality for $\left|Y_{1}\right|+\left|Y_{2}\right| \leq 6$.

${ }^{16}$ We also checked this equality for $\left|Y_{1}\right|+\left|Y_{2}\right| \leq 6$.
} 
Note that, depending on how the $\mathrm{U}(1)$-part of the gauge group couples to $\left(A_{1}, D_{4}\right)$, its contribution to the partition function is $\mathcal{Z}_{Y_{1}, Y_{2}}^{\left(A_{1}, D_{4}\right)}$ or $\widetilde{\mathcal{Z}}_{Y_{1}, Y_{2}}^{\left(A_{1}, D_{4}\right)}$. In this section, we focus on the case in which both of the two $\left(A_{1}, D_{4}\right)$ theories couple to the $\mathrm{U}(1)$ in the way corresponding to $\mathcal{Z}_{Y_{1}, Y_{2}}^{\left(A_{1}, D_{4}\right)}$. Replacing one or two of $\mathcal{Z}_{Y_{1}, Y_{2}}^{\left(A_{1}, D_{4}\right)}$ with $\widetilde{\mathcal{Z}}_{Y_{1}, Y_{2}}^{\left(A_{1}, D_{4}\right)}$, one would obtain to a different $\mathcal{Z}_{\mathrm{U}(2)}$, which is however expected to give the same $\mathcal{Z}_{\mathrm{SU}(2)}$ when the $\mathrm{U}(1)$ factor $\mathcal{Z}_{\mathrm{U}(1)}$ is removed as in (1.4).

The factor $\mathcal{Z}_{Y_{1}, Y_{2}}^{\left(A_{1}, D_{4}\right)}$ in eq. (5.1) is given by

$$
\mathcal{Z}_{Y_{1}, Y_{2}}^{\left(A_{1}, D_{4}\right)}(a, m, d, u)=\left(-\frac{c_{2}}{2}\right)^{-\frac{\left|Y_{1}\right|+\left|Y_{2}\right|}{2}} \frac{\left\langle a ; Y_{1}, Y_{2} \mid \widehat{I}^{(2)}\right\rangle}{\left\langle a \mid \widehat{I}^{(2)}\right\rangle},
$$

where $m, d$ and $u$ are identified as

$$
m=2 c_{0}+\frac{c_{1}^{2}}{c_{2}}, \quad d=\frac{2 c_{1}}{\sqrt{c_{2}}}, \quad u=\frac{2 c_{0} c_{1}}{\sqrt{c_{2}}}-\sqrt{c_{2}} \frac{\partial \mathcal{F}_{\left(A_{1}, D_{4}\right)}}{\partial c_{1}},
$$

with $\mathcal{F}_{\left(A_{1}, D_{4}\right)}$ being the classical limit of $\log \left\langle a \mid \widehat{I}^{(2)}\right\rangle$. Note that we here set $\zeta=-1 / 2$ in (4.12) to avoid various numerical factors in the expressions below. This factor can be generated or absorbed by rescaling $q$ in the expression (5.1). The irregular state $\left|\widehat{I}^{(2)}\right\rangle$ is characterized by

$$
\begin{aligned}
L_{4}\left|\widehat{I}^{(2)}\right\rangle & =-c_{2}^{2}\left|\widehat{I}^{(2)}\right\rangle, \\
L_{3}\left|\widehat{I}^{(2)}\right\rangle & =-2 c_{1} c_{2}\left|\widehat{I}^{(2)}\right\rangle, \\
L_{2}\left|\widehat{I}^{(2)}\right\rangle & =-\left(c_{1}^{2}+c_{2}\left(2 c_{0}-3 Q\right)\right)\left|\widehat{I}^{(2)}\right\rangle, \\
L_{1}\left|\widehat{I}^{(2)}\right\rangle & =\left(c_{2} \frac{\partial}{\partial c_{1}}-2 c_{1}\left(c_{0}-Q\right)\right)\left|\widehat{I}^{(2)}\right\rangle, \\
L_{0}\left|\widehat{I}^{(2)}\right\rangle & =\left(\Delta_{c_{0}}+c_{1} \frac{\partial}{\partial c_{1}}+2 c_{2} \frac{\partial}{\partial c_{2}}\right)\left|\widehat{I}^{(2)}\right\rangle,
\end{aligned}
$$

and $a_{k}\left|\widehat{I}^{(2)}\right\rangle=-i c_{k}\left|\widehat{I}^{(2)}\right\rangle$ for $k=1,2$ together with $a_{k}\left|\widehat{I}^{(2)}\right\rangle=0$ for $k>2$.

To extract the partition function of the $\left(A_{3}, A_{3}\right)$ theory from (5.1), one has to remove the contribution of the U(1)-part of the gauge group. As discussed in section 1, this can be done by dividing (5.1) by a $\mathrm{U}(1)$ factor, $\mathcal{Z}_{\mathrm{U}(1)}$. Therefore the partition function of the $\left(A_{3}, A_{3}\right)$ theory is identified as

$$
\mathcal{Z}_{\left(A_{3}, A_{3}\right)}=\frac{\mathcal{Z}_{\mathrm{U}(2)}}{\mathcal{Z}_{\mathrm{U}(1)}}
$$

While it is beyond the scope of this paper to determine the $\mathrm{U}(1)$ factor, we know that $\mathcal{Z}_{\mathrm{U}(1)}$ is independent of the parameter $a$, since $a$ is the $\mathrm{VEV}$ of a scalar in the $\mathrm{SU}(2)$ vector multiplet that is neutral under the $\mathrm{U}(1)$. Below, we use this fact and compute the classical limit of $\mathcal{Z}_{\left(A_{3}, A_{3}\right)}$.

\subsection{Prepotential}

Here we consider the classical limit $\epsilon_{i} \rightarrow 0$, and compute the prepotential of the $\left(A_{3}, A_{3}\right)$ theory

$$
\mathcal{F}^{\left(A_{3}, A_{3}\right)} \equiv \lim _{\epsilon_{i} \rightarrow 0}\left(-\epsilon_{1} \epsilon_{2} \log \mathcal{Z}_{\left(A_{3}, A_{3}\right)}\right)
$$


This prepotential splits into the perturbative and instanton parts as $\mathcal{F}^{\left(A_{3}, A_{3}\right)}=\mathcal{F}_{\text {pert }}^{\left(A_{3}, A_{3}\right)}+$ $\mathcal{F}_{\text {inst }}^{\left(A_{3}, A_{3}\right)}$, and we are particularly interested in the instanton part $\mathcal{F}_{\text {inst }}^{\left(A_{3}, A_{3}\right)}{ }^{17}$ The instanton part is generally expanded as

$$
\mathcal{F}_{\text {inst }}^{\left(A_{3}, A_{3}\right)}=\sum_{k=1}^{\infty} \mathcal{F}_{k} q^{k} .
$$

Below, we will compute the coefficients, $\mathcal{F}_{k}$, in this expansion.

To that end, let us first consider

$$
\mathcal{F}^{\mathrm{U}(2)} \equiv \lim _{\epsilon_{i} \rightarrow 0}\left(-\epsilon_{1} \epsilon_{2} \log \mathcal{Z}_{\mathrm{U}(2)}\right)
$$

which is the prepotential of the theory with the gauge group replaced by $\mathrm{U}(2)$. This prepotential also splits into the perturbative part, $\lim _{\epsilon_{i} \rightarrow 0}\left(-\epsilon_{1} \epsilon_{2} \log \mathcal{Z}_{\text {pert }}^{\mathrm{U}(2)}\right)$, and the instanton part

$$
\mathcal{F}_{\text {inst }}^{\mathrm{U}(2)} \equiv \lim _{\epsilon_{i} \rightarrow 0}\left(-\epsilon_{1} \epsilon_{2} \log \frac{\mathcal{Z}^{\mathrm{U}(2)}}{\mathcal{Z}_{\text {pert }}^{\mathrm{U}(2)}}\right)
$$

The instanton part (5.13) is identical to $\mathcal{F}_{\text {inst }}^{\left(A_{3}, A_{3}\right)}$ up to the contribution of the $\mathrm{U}(1)$ factor. Our strategy is to compute $\mathcal{F}_{\text {inst }}^{\mathrm{U}(2)}$ using the formula (5.1), and then strip off the $\mathrm{U}(1)$ factor to obtain $\mathcal{F}_{\text {inst }}^{\left(A_{3}, A_{3}\right)}$.

Note that the computation of $\mathcal{Z}_{\mathrm{U}(2)}$ via (5.1) and (5.2) eventually reduces to evaluating

$$
\left\langle a\left|L_{k_{p}}^{m_{p}} \cdots L_{k_{1}}^{m_{1}} a_{\ell_{q}}^{n_{q}} \cdots a_{\ell_{1}}^{n_{1}}\right| \widehat{I}^{(2)}\right\rangle,
$$

for positive integers $k_{i}, m_{i}, \ell_{j}$ and $n_{j}$. Using (5.4)-(5.6) and the fact that $L_{k}\left|\widehat{I}^{(2)}\right\rangle=$ $a_{\ell}\left|\widehat{I}^{(2)}\right\rangle=0$ for $k>4$ and $\ell>2$, this computation further reduces to evaluating

$$
\left\langle a\left|L_{1}^{k}\right| \widehat{I}^{(2)}\right\rangle=\left(c_{2} \frac{\partial}{\partial c_{1}}-2 c_{1}\left(c_{0}-Q\right)\right)^{k}\left\langle a \mid \widehat{I}^{(2)}\right\rangle
$$

where $\left\langle a \mid \widehat{I}^{(2)}\right\rangle$ is the partition function of $\left(A_{1}, D_{4}\right)$ theory (with its flavor symmetry ungauged). Therefore, to compute $\mathcal{Z}_{\mathrm{U}(2)}$ for general $\Omega$-background parameters, one needs to know how $\left\langle a \mid \widehat{I}^{(2)}\right\rangle$ depends on $c_{1} \cdot{ }^{18}$ However, in the classical limit $\epsilon_{i} \rightarrow 0$, one can skip this procedure. Indeed, recovering the full $\epsilon_{i}$-dependence by $c_{k} \rightarrow c_{k} / \sqrt{\epsilon_{1} \epsilon_{2}}$, we see that in the classical limit

$$
\left\langle a\left|L_{1}^{k}\right| \widehat{I}^{(2)}\right\rangle=\left(c_{2}\right)^{\frac{k}{2}}(-u)^{k}\left\langle a \mid \widehat{I}^{(2)}\right\rangle,
$$

where $u$ is defined by eq. (5.3). Given (5.4)-(5.6) and (5.16), it is straightforward to compute the classical limit of $\mathcal{Z}_{\mathrm{U}(2)}$, and therefore $\mathcal{F}_{\text {inst }}^{\mathrm{U}(2)}$, order by order in $q$.

\footnotetext{
${ }^{17}$ Note that the perturbative part contains the prepotential of the $\left(A_{1}, D_{4}\right)$ theories (with their flavor symmetries ungauged).

${ }^{18}$ The $1 / c_{1}$-expansion of $\left\langle a \mid I^{(2)}\right\rangle$ was carefully studied in [30].
} 
We now turn to the $\mathrm{U}(1)$ factor. While it is generically non-vanishing, the contribution from the $\mathrm{U}(1)$-factor turns out to vanish when all the dimensionful parameters in fourdimensions, except for $a$ and $\epsilon_{i}$, are turned off. Indeed, in the classical limit, $\mathcal{Z}_{\mathrm{U}(1)} \sim$ $\exp \left(-\frac{1}{\epsilon_{1} \epsilon_{2}} \mathcal{F}_{\mathrm{U}(1)}\right)$ with $\mathcal{F}_{\mathrm{U}(1)}$ being independent of $\epsilon_{i}$. When dimensionful parameters are turned off except for $a$ and $\epsilon_{i}, \mathcal{F}_{\mathrm{U}(1)}$ must be proportional to $a^{2}$ for dimensional reasons. However, as discussed below (5.9), $\mathcal{Z}_{\mathrm{U}(1)}$ must be independent of $a$. This means that the proportionality constant is zero so that $\mathcal{F}_{\mathrm{U}(1)}=0$.

Let us now focus on the case in which $M, m_{i}, d_{i}$ and $u_{i}$ in (5.1) are all turned off. Then the only non-vanishing dimensionful parameters are $a$ and $\epsilon_{i}$. Since the U(1)-factor is trivial in this case, one can identify (5.10) with (5.12), and therefore (5.11) with (5.13). With this identification, we finally obtain

$$
\mathcal{F}_{\text {inst }}^{\left(A_{3}, A_{3}\right)}(q ; a)=\left(\frac{1}{4} q^{2}+\frac{13}{128} q^{4}+\frac{23}{384} q^{6}+\frac{2701}{65536} q^{8}+\cdots\right) a^{2} .
$$

Remarkably, this expression is closely related to the instanton part of the prepotential of the $\mathrm{SU}(2)$ gauge theory with four fundamental flavors. Indeed, when all the mass parameters are turned off, the latter is given by

$$
\mathcal{F}_{\text {inst }}^{N_{f}=4}(q ; a)=\left(\frac{1}{2} q+\frac{13}{64} q^{2}+\frac{23}{192} q^{3}+\frac{2701}{32768} q^{4}+\cdots\right) a^{2},
$$

as shown in appendix B.3 of [19]. Comparing (5.17) and (5.18), we see that

$$
2 \mathcal{F}_{\text {inst }}^{\left(A_{3}, A_{3}\right)}(q ; a)=\mathcal{F}_{\text {inst }}^{N_{f}=4}\left(q^{2}, a\right),
$$

at least up to $\mathcal{O}\left(q^{8}\right)$.

\section{$5.2 \quad$ S-duality}

Here, we show that one can read off the action of the S-duality group on the $\left(A_{3}, A_{3}\right)$ theory assuming the remarkable identity (5.19) extends to the full prepotential. To that end, let us first give a quick review of the S-duality of $\mathrm{SU}(2)$ gauge theory with four fundamental flavors. When the mass parameters are turned off, the full prepotential of this theory is written as

$$
\mathcal{F}^{N_{f}=4}=\left(\log q_{\mathrm{IR}}\right) a^{2}
$$

where $q_{\mathrm{IR}}$ is related to the IR theta angle and electric coupling by

$$
q_{\mathrm{IR}}=e^{i \theta_{\mathrm{IR}}-\frac{8 \pi^{2}}{g_{\mathrm{IR}}^{2}}} .
$$

The full prepotential (5.20) is the sum of the instanton part (5.18) and the perturbative part $(\log q-\log 16) a^{2}$. This implies that $q$ and $q_{\mathrm{IR}}$ are related by [36]

$$
q=\frac{\theta_{2}\left(q_{\mathrm{IR}}\right)^{4}}{\theta_{3}\left(q_{\mathrm{IR}}\right)^{4}}
$$


where we used the convention that $\theta_{2}(q)=\sum_{n \in \mathbb{Z}} q^{\left(n-\frac{1}{2}\right)^{2}}$ and $\theta_{3}(q)=\sum_{n \in \mathbb{Z}} q^{n^{2}}$. The relation (5.22) implies that

$$
\tau_{\mathrm{IR}} \equiv \frac{1}{\pi i} \log q_{\mathrm{IR}}=\frac{\theta_{\mathrm{IR}}}{\pi}+\frac{8 \pi i}{g_{\mathrm{IR}}^{2}}
$$

is the modulus of the elliptic curve corresponding to the double cover of $\mathbb{C} \mathbb{P}^{1}$ with four branch points whose cross-ratio is $q$. This elliptic curve is identified as the SW-curve of the theory on the Coulomb branch. The curve has a natural $\operatorname{PSL}(2, \mathbb{Z})$-action generated by

$$
T: \tau_{\mathrm{IR}} \rightarrow \tau_{\mathrm{IR}}+1, \quad S: \tau_{\mathrm{IR}} \rightarrow-\frac{1}{\tau_{\mathrm{IR}}}
$$

From (5.22), we see that these $T$ and $S$ transformations are induced by the following changes of the UV gauge couplings:

$$
T: q \rightarrow \frac{q}{q-1}, \quad S: q \rightarrow 1-q
$$

Since the SW-curve is invariant under $T$ and $S$, so is the whole BPS spectrum on the Coulomb branch. It is then natural to expect that the theory is completely invariant under this $\operatorname{PSL}(2, \mathbb{Z}) .{ }^{19}$ This is the famous S-duality of $\mathrm{SU}(2)$ gauge theory with four flavors.

Let us now turn back to the $\left(A_{3}, A_{3}\right)$ theory. Its quiver description shown in figure 1 has an obvious similarity to the $\mathrm{SU}(2)$ gauge theory with four flavors; it has the same gauge group with the vanishing $\beta$-function. This similarity has been studied carefully in [6-8] to show that the IR physics of the $\left(A_{3}, A_{3}\right)$ theory on its Coulomb branch admits an action of $\operatorname{PSL}(2, \mathbb{Z})$ (see [9-13] for further studies on this new class of $\mathcal{N}=2$ S-dualities). The generalization of the $\mathrm{SO}(8)$-triality in this case is carefully discussed in [8]. This $\operatorname{PSL}(2, \mathbb{Z})$-action has only been studied in the IR language such as the SW curve, the associated Calabi-Yau three-fold, and the spectrum of BPS states on the Coulomb branch. Here we discuss the action of $\operatorname{PSL}(2, \mathbb{Z})$ on the UV gauge coupling, using the surprising relation (5.19).

To that end, let us define the IR gauge coupling $q_{\mathrm{IR}}$ of the $\left(A_{3}, A_{3}\right)$ theory similarly by

$$
q_{\mathrm{IR}}=e^{i \theta_{\mathrm{IR}}-\frac{8 \pi^{2}}{g_{\mathrm{IR}}^{2}}}
$$

so that the full prepotential is written as

$$
\mathcal{F}^{\left(A_{3}, A_{3}\right)}=\left(\log q_{\mathrm{IR}}\right) a^{2}
$$

\footnotetext{
${ }^{19}$ When mass parameters are turned on, they are also permuted by the action of PSL $(2, \mathbb{Z})$.
} 
Assuming the relation (5.19) extends to the full prepotential, one obtains ${ }^{20}$

$$
2 \mathcal{F}^{\left(A_{3}, A_{3}\right)}(q ; a)=\mathcal{F}^{N_{f}=4}\left(q^{2} ; a\right),
$$

which implies that $q_{\mathrm{IR}}$ is related to $q$ by

$$
q^{2}=\frac{\theta_{2}\left(q_{\mathrm{IR}}^{2}\right)^{4}}{\theta_{3}\left(q_{\mathrm{IR}}^{2}\right)^{4}}
$$

This relation means that

$$
\tau_{\mathrm{IR}} \equiv \frac{2}{\pi i} \log q_{\mathrm{IR}}=\frac{2 \theta_{\mathrm{IR}}}{\pi}+\frac{16 \pi i}{g_{\mathrm{IR}}^{2}}
$$

is the modulus of the double cover of $\mathbb{C P}^{1}$ with four branch points whose cross-ratio is $q^{2}$. The $T$ and $S$ transformations of the S-duality group are identified as

$$
T: \tau_{\mathrm{IR}} \rightarrow \tau_{\mathrm{IR}}+1, \quad S: \tau_{\mathrm{IR}} \rightarrow-\frac{1}{\tau_{\mathrm{IR}}} .
$$

Note that (5.31) reveals a non-trivial relation between the IR gauge coupling $q_{\mathrm{IR}}$ and the UV gauge coupling $q$ of the $\left(A_{3}, A_{3}\right)$ theory. Compared to (5.22) for $\mathrm{SU}(2)$ gauge theory with four flavors, both the UV and IR gauge couplings are replaced by their squares here. While the replacement $q \rightarrow q^{2}$ can be easily understood from the relation (5.30), the replacement

$$
q_{\mathrm{IR}} \rightarrow q_{\mathrm{IR}}^{2}
$$

is a bit more non-trivial. We see that this replacement is a consequence of the factor $2 \mathrm{in}$ front of $\mathcal{F}^{\left(A_{3}, A_{3}\right)}$ in (5.30). Note that (5.34) is crucial to have the correct weak-coupling behavior. Indeed, in the weak coupling limit, quantum corrections to the IR gauge coupling vanish, and therefore we expect $q=q_{\mathrm{IR}}$. We see that (5.31) correctly reduces to $q \sim q_{\mathrm{IR}}$ in the weak coupling limit $q_{\mathrm{IR}} \rightarrow 0$ if (5.34) is simultaneously performed with $q \rightarrow q^{2}$.

From the above discussion, we see how $S$ and $T$ act on the UV gauge coupling of the $\left(A_{3}, A_{3}\right)$ theory. Indeed, combining (5.31) and (5.32), we see that (5.33) corresponds to

$$
T: q^{2} \rightarrow \frac{q^{2}}{q^{2}-1}, \quad S: q^{2} \rightarrow 1-q^{2}
$$

One can explicitly check that (5.17) combined with the classical part $\mathcal{F}_{\mathrm{cl}}^{\left(A_{3}, A_{3}\right)}=(\log q) a^{2}$ is invariant under $T$.

\footnotetext{
${ }^{20}$ Given the relation (5.19) for the instanton part, this assumption is rather mild. Indeed, it only requires that the perturbative part $\mathcal{F}_{\text {pert }}=\mathcal{F}_{\text {cl }}+\mathcal{F}_{1 \text {-loop }}$ also satisfies

$$
2 \mathcal{F}_{\text {pert }}^{\left(A_{3}, A_{3}\right)}(q ; a)=\mathcal{F}_{\text {pert }}^{N_{f}=4}\left(q^{2} ; a\right) .
$$

Note that, on both sides of the above relation, $\mathcal{F}_{\text {pert }}=(\log q+X) a^{2}$ with $X$ being a constant. Therefore, in proving (5.28), all we need to show is the following equality between two constants:

$$
2 X^{\left(A_{3}, A_{3}\right)}=X^{N_{f}=4} .
$$

To prove this, one needs to identify the 1-loop part $\mathcal{F}_{1 \text {-loop }}$ for $\left(A_{3}, A_{3}\right)$, which we leave for future work.
} 


\subsection{Peculiarity of $T$}

From (5.32), we see that our $T$-transformation, $\tau_{\mathrm{IR}} \rightarrow \tau_{\mathrm{IR}}+1$, corresponds to

$$
\theta_{\mathrm{IR}} \rightarrow \theta_{\mathrm{IR}}+\frac{\pi}{2}
$$

This is remarkable since this means that $T$ maps the monopole of the minimal magnetic charge to a dyon that has half the electric charge of fundamental quark. This is not possible if this "electric charge" is a charge associated with the unbroken $\mathrm{U}(1) \subset \mathrm{SU}(2)$ gauge group on the Coulomb branch, since in that case the minimal electric charge is the charge of fundamental quark. Therefore, the "electric charge" here is not simply associated with the unbroken $\mathrm{U}(1) \subset \mathrm{SU}(2)$. As we will argue in section 6 , the "electric charge" here is interpreted as a linear combination of the electric charge associated with $\mathrm{U}(1) \subset \mathrm{SU}(2)$ and those arising from the $\left(A_{1}, D_{4}\right)$ theories.

In the rest of this sub-section, we show that the $T$ transformation (5.36) is also consistent with the SW curve of $\left(A_{3}, A_{3}\right)$ theory. To that end, first recall that the SW curve of this theory is written as [6]

$$
0=x^{4}+\mathrm{q} x^{2} z^{2}+z^{4}+c_{3,0} x^{3}+c_{0,3} z^{3}+c_{2,0} x^{2}+m x z+c_{0,2} z^{2}+c_{1,0} x+c_{0,1} z+c_{0,0},
$$

where $\mathrm{q}$ is a function of $q_{\mathrm{IR}}, c_{3,0}$ and $c_{0,3}$ are relevant couplings of dimension $1 / 2, c_{2,0}, c_{0,2}$ and $m$ are mass parameters, and $c_{1,0}, c_{0,1}$ and $c_{0,0}$ parameterize the Coulomb branch moduli space. The SW 1-form is given by $x d z$. It was shown in [6] that the above curve and 1-form are invariant under two transformations $\widetilde{S}$ and $\widetilde{T}$, which were interpreted as two independent S-dual transformations. In particular, $\widetilde{S}$ acts on the marginal gauge coupling as ${ }^{21}$

$$
\widetilde{S}: \mathrm{q} \rightarrow-\mathrm{q} .
$$

Below, we show that (5.38) is identical to our $T$-transformation (5.36), near a cusp on the conformal manifold. ${ }^{22}$ As shown in [6], $q \rightarrow \infty$ corresponds to a weak-coupling cusp on the conformal manifold, where $M \equiv m / q$ and $u \equiv c_{0,0} / \mathrm{q}$ are respectively identified as the mass of the fundamental hypermultiplet and the VEV of the Coulomb branch operator in the vector multiplet, in the quiver description in figure $1 .{ }^{23}$ We focus on this cusp, and turn on an infinitely large mass, $M$, for the fundamental hypermultiplet so that it decouples from the theory in the infrared. When the hypermultiplet decouples, the theory reduces to a non-conformally gauged AD theory described in figure 2. To realize this limit at the level of the SW curve, one needs to take $\mathrm{q} \rightarrow \infty$ simultaneously with $M \rightarrow \infty$. Indeed, if we take $M \rightarrow \infty$ with q kept fixed, some periods of the curve are divergent. It turns out

\footnotetext{
${ }^{21}$ The $\widetilde{T}$-transformation acts on the gauge coupling as $\widetilde{T}: \mathrm{q} \rightarrow \frac{12-2 \mathrm{q}}{2+\mathrm{q}}$. Our $T$ and $S$ correspond to $\widetilde{S}$ and $\widetilde{T}$ in $[6]$, respectively.

${ }^{22}$ Here, the "conformal manifold" is defined as the space of values of exactly marginal couplings in the theory.

${ }^{23}$ There are also other cusps at $\mathrm{q} \rightarrow \pm 2$, where the parameters in the SW curve have different interpretations. In particular, the mass of the fundamental hypermultiplet is identified with some linear combination of $m, c_{2,0}$ and $c_{0,2}$.
} 
that all the periods of the curve remain finite when one takes $M \rightarrow \infty$ and $\mathrm{q} \rightarrow \infty$ with

$$
\Lambda \equiv \frac{M}{\sqrt{\mathrm{q}}}
$$

kept fixed. This $\Lambda$ is then identified as a dynamical scale of the resulting theory. The curve correctly reduces to the curve of the IR theory (4.17) (for $n=2$ ) in the limit $M, q \rightarrow \infty$ with (5.39) kept finite. ${ }^{24}$

Note here that, by standard arguments, the dynamical scale $\Lambda$ of the mass-deformed theory and the gauge coupling $e^{i \theta_{\mathrm{IR}}-\frac{8 \pi^{2}}{g_{\mathrm{IR}}^{2}}}$ of the conformal theory are related by

$$
\left(\frac{\Lambda}{M}\right)^{b_{0}}=e^{i \theta_{\mathrm{IR}}-\frac{8 \pi^{2}}{g_{\mathrm{IR}}^{2}}}
$$

where $b_{0}$ is the coefficient of the one-loop $\beta$-function of the IR theory. Since $b_{0}=1$ in our case, (5.39) and (5.41) imply that

$$
\mathbf{q}=e^{-2 i \theta_{\mathrm{IR}}+\frac{16 \pi^{2}}{g_{\mathrm{IR}}^{2}}}
$$

From this relation, it is now clear that the $\widetilde{S}$ transformation (5.38) is precisely identical to our $T$-transformation (5.36). Therefore, our $T$-transformation (5.36) is perfectly consistent with the earlier analysis of the S-duality using the SW-curve.

\subsection{Turning on couplings, masses and VEVs}

In the previous sections, we have identified the $\operatorname{PSL}(2, \mathbb{Z})$-action in the case of vanishing dimensionful parameters except for $\epsilon_{i}$ and $a$. This action has been interpreted as corresponding to the symmetry of the SW-curve studied in [6]. Since this symmetry of the curve extends to the case of generic values of dimensionful parameters, we expect that the PSL $(2, \mathbb{Z})$-action on the partition function has a similar extension for non-vanishing relevant couplings, masses and VEVs of Coulomb branch operators. In this sub-section, we discuss such an extension of the $\operatorname{PSL}(2, \mathbb{Z})$-action.

\footnotetext{
${ }^{24}$ One can show this explicitly as follows. As show in [6], near the cusp $\mathrm{q} \rightarrow \infty, C_{3,0} \equiv \mathrm{q}^{-\frac{1}{4}} c_{3,0}$ and $C_{0,3} \equiv \mathrm{q}^{-\frac{1}{4}} c_{0,3}$ are identified with the (correctly-normalized) relevant couplings of dimension $1 / 2, C_{2,0} \equiv$ $\mathrm{q}^{-\frac{1}{2}} c_{2,0}$ and $C_{0,2} \equiv \mathrm{q}^{-\frac{1}{2}} c_{0,2}$ are mass deformation parameters, and $C_{1,0} \equiv \mathrm{q}^{-\frac{3}{2}} c_{1,0}$ and $C_{0,1} \equiv \mathrm{q}^{-\frac{3}{4}} c_{0,1}$ are the VEVs of Coulomb branch operators, in the $\left(A_{1}, D_{4}\right)$ sectors. In terms of these variables, the curve of the $\left(A_{3}, A_{3}\right)$ theory is written as $0=x^{4}+\mathrm{q} x^{2} z^{2}+z^{4}+\mathrm{q}^{\frac{1}{4}} C_{3,0} x^{3}+\mathrm{q}^{\frac{1}{4}} C_{0,3} z^{3}+\mathrm{q}^{\frac{1}{2}} C_{2,0} x^{2}+\mathrm{q} M x z+\mathrm{q}^{\frac{1}{2}} C_{0,2} z^{2}+$ $\mathrm{q}^{\frac{3}{4}} C_{1,0} x+\mathrm{q}^{\frac{3}{4}} C_{0,1} z+\mathrm{q} u$. We here define $X \equiv i\left(\sqrt{z} x^{\frac{3}{2}}+\frac{1}{2} \sqrt{q} \Lambda \sqrt{x / z}\right), Z \equiv \sqrt{z / x}$ and $U \equiv u-\frac{q \Lambda^{2}}{4}$, and take the limit $M, \mathrm{q} \rightarrow \infty$ with $\Lambda, U, C_{i, j}$ and $(X, Y)$ kept finite. Then the curve reduces to

$$
X^{2}=\widetilde{\Lambda}^{2} Z^{2}+\widetilde{\Lambda}^{\frac{3}{2}} C_{0,3} Z+\widetilde{\Lambda} C_{0,2}+\frac{\widetilde{\Lambda}^{\frac{1}{2}} C_{0,1}}{Z}+\frac{U}{Z^{2}}+\frac{\widetilde{\Lambda}^{\frac{1}{2}} C_{1,0}}{Z^{3}}+\frac{\widetilde{\Lambda} C_{2,0}}{Z^{4}}+\frac{\widetilde{\Lambda}^{\frac{3}{2}} C_{3,0}}{Z^{5}}+\frac{\widetilde{\Lambda}^{2}}{Z^{6}},
$$

where $\widetilde{\Lambda} \equiv-\Lambda / 2$. The SW 1 -form is written as $-\frac{3}{2} i X d Z$ up to exact terms. This curve is precisely identical to (4.17) for $n=2$. The 1 -form is also identical up to a prefactor that can be absorbed by rescaling dimensionful parameters and $X$. This implies that (5.39) is the correct identification of the IR dynamical scale.
} 
The instanton part of the prepotential for generic values of dimensionful parameters is expanded as

$$
\mathcal{F}_{\text {inst }}^{\left(A_{3}, A_{3}\right)}=\sum_{k=-1}^{\infty} \mathcal{F}_{2 k}\left(q, M,\left\{m_{i}\right\},\left\{u_{i}\right\},\left\{d_{i}\right\}\right) a^{-2 k}
$$

Recall that $T$ and $S$ act on the UV gauge coupling as in (5.35). Since $S$ is non-perturbative in $q$, it is hard to understand how (5.43) behaves under $S$ using our order-by-order computation. We therefore focus on the $T$-transformation below.

In the case of $d_{i}=m_{i}=u_{i}=M=0,(5.43)$ reduces to

$$
\mathcal{F}_{-2}(q) a^{2}
$$

whose explicit expression is shown in eq. (5.17). When combined with the perturbative part, this is invariant under $T: q^{2} \rightarrow q^{2} /\left(q^{2}-1\right)$.

When $d_{i}, m_{i}, u_{i}$ and $M$ are turned on, (5.44) receives corrections from $\mathcal{F}_{2 k}$ for all $k \geq 0$. We evaluated these corrections using our formula (5.2) to find that $\mathcal{F}_{-2}$ and $\mathcal{F}_{k>0}$ are all invariant under

$$
q \rightarrow \frac{i q}{\sqrt{1-q^{2}}}, \quad d_{1} \rightarrow \frac{d_{1}+q d_{2}}{\sqrt{1-q^{2}}}, \quad d_{2} \rightarrow i d_{2}, \quad m_{2} \rightarrow-m_{2}, \quad u_{2} \rightarrow-i u_{2},
$$

with the other parameters kept fixed. ${ }^{25}$ We checked this invariance up to a very high order of $q$. First few terms in the $q$-series of $\mathcal{F}_{-2}, \mathcal{F}_{2}$ and $\mathcal{F}_{4}$ are shown in appendix C. Given this invariance, we interpret (5.45) as an extension of $q^{2} \rightarrow q^{2} /\left(q^{2}-1\right)$ to the case of non-vanishing $d_{i}, m_{i}, u_{i}$ and $M$.

It turns out that (5.45) is consistent with the symmetry of the SW curve, at least in the weak coupling limit. To see this, recall that our $T$-transformation is identified with $\widetilde{S}$ discussed in [6]. This $\widetilde{S}$ induces $\mathrm{q} \rightarrow-\mathrm{q}$, as reviewed already, and also changes the other parameters in the SW curve as

$$
\begin{array}{clll}
c_{3,0} \rightarrow-e^{\frac{3 \pi i}{4}} c_{3,0}, & c_{0,3} \rightarrow-e^{-\frac{3 \pi i}{4}} c_{0,3}, & c_{2,0} \rightarrow-i c_{2,0}, & c_{0,2} \rightarrow i c_{0,2}, \\
m \rightarrow-m, & c_{1,0} \rightarrow-e^{\frac{\pi i}{4}} c_{1,0}, & c_{0,1} \rightarrow-e^{-\frac{\pi i}{4}} c_{0,1}, & c_{0,0} \rightarrow-c_{0,0} .
\end{array}
$$

It is straightforward to show that the curve (5.37) and the SW 1-form is invariant under these transformations. Since $q^{2} \rightarrow q^{2} /\left(q^{2}-1\right)$ is already identified with $\mathbf{q} \rightarrow-\mathbf{q},(5.46)$ is expected to be equivalent to (5.45). One can show this equivalence explicitly at the weak-coupling cusp $q=\infty$. As shown in [6], in the limit $q \rightarrow \infty$, the coefficients $c_{i, j}$ must be appropriately renormalized so that physical quantities remain finite. In terms of our $d_{i}, m_{i}, u_{i}, M$ and $u$, such a renormalization is expressed as

$$
\begin{aligned}
& c_{3,0}=\mathrm{q}^{\frac{1}{4}} d_{1}, \quad c_{0,3}=\mathrm{q}^{\frac{1}{4}} d_{2}, \quad c_{2,0}=\mathrm{q}^{\frac{1}{2}} m_{1}, \quad c_{0,2}=\mathrm{q}^{\frac{1}{2}} m_{2}, \quad m=\mathrm{q} M ， \\
& c_{1,0}=\mathrm{q}^{\frac{3}{4}} u_{1}, \quad c_{0,1}=\mathrm{q}^{\frac{3}{4}} u_{2}, \quad c_{0,0}=\mathrm{q} u .
\end{aligned}
$$

\footnotetext{
${ }^{25}$ Note that $\mathcal{F}_{0}$ cannot be evaluated without identifying the $\mathrm{U}(1)$-factor, and therefore we leave the computation of $\mathcal{F}_{0}$ for future work. The other terms, $\mathcal{F}_{-2}$ and $\mathcal{F}_{k>0}$, are not affected by the U(1)-factor.
} 
From (5.47) and (5.46), we see that $\widetilde{S}$ is equivalent to $\mathrm{q} \rightarrow \exp (-\pi i) \mathrm{q}$ combined with

$$
d_{2} \rightarrow i d_{2}, \quad m_{2} \rightarrow-m_{2}, \quad u_{2} \rightarrow-i u_{2},
$$

where $d_{1}, m_{1}, u_{1}, M$ and $u$ are kept fixed. Since $\mathrm{q} \rightarrow \exp (-\pi i) \mathrm{q}$ is identified with $q^{2} \rightarrow$ $q^{2} /\left(q^{2}-1\right)$, this is identical to (5.45) at the leading order of $q$. The reason that we only see the leading order terms is that we are taking the weak-coupling limit $\mathrm{q} \rightarrow \infty$ here, corresponding to $q \rightarrow 0$. Thus, we have shown that our (5.45) is equivalent to (5.46) in the weak-coupling limit.

Note that the invariance of $\mathcal{F}_{\text {inst }}^{\left(A_{3}, A_{3}\right)}$ under (5.45) strongly constrains the possible form of $\mathcal{F}_{k>0}$. In particular, when combined with the trivial symmetry under exchanging two $\left(A_{1}, D_{4}\right)$ theories in the quiver diagram, imposing this invariance determines $\mathcal{F}_{k>0}$ up to some $T$-invariant functions for every $k$. We explicitly show this for $\mathcal{F}_{2}$ in appendix C.

Note also that our check of the invariance under (5.45) is only for the instanton part of the prepotential. For the full prepotential to be invariant under (5.45), the perturbative part must also be invariant by itself. One can show that the perturbative part is invariant under (5.45) when $q=0$, but its extension to the case of $q \neq 0$ is not straightforward. If (5.45) also preserves the perturbative part for general $q$, it is natural to identify (5.45) as the $T$-transformation for generic values of $d_{i}, m_{i}$ and $u_{i}$. If the invariance under (5.45) does not extend to the perturbative part, (5.45) is a new symmetry that only preserves the instanton part. We leave a detailed study of these two options for future work.

\section{Conclusions and discussions}

In this paper, we have proposed a Nekrasov-type formula for the instanton partition function of four-dimensional $\mathcal{N}=2 \mathrm{U}(2)$ gauge theories coupled to $\left(A_{1}, D_{2 n}\right)$ Argyres-Douglas theories, by extending the generalized AGT correspondence to the case of $\mathrm{U}(2)$ gauge group. We have defined irregular states of the direct sum of Virasoro and Heisenberg algebras, and then identified the contribution of the $\left(A_{1}, D_{2 n}\right)$ theory at each fixed points on the $\mathrm{U}(2)$ instanton moduli space, as in (4.12) and (4.13). Here, $4 \mathrm{~d}$ and $2 \mathrm{~d}$ parameters are related by (4.9).

As an application, we evaluate the instanton partition function of the $\left(A_{3}, A_{3}\right)$ theory. While this partition function cannot be directly evaluated by the AGT correspondence, we have computed it using our formula above. Our result shows that, when some parameters are turned off, the instanton part of the prepotential of $\left(A_{3}, A_{3}\right)$ is in a surprising relation to that of $\mathrm{SU}(2)$ gauge theory with four flavors, as shown in (5.19). In particular, the two prepotentials are related by the following replacement of the UV gauge coupling:

$$
q \rightarrow q^{2} .
$$

From this relation, we have read off the action of the S-duality group on the UV gauge coupling $q$. We have also discussed its possible extension to dimensionful parameters.

Here, we give a brief comment on our peculiar $T$-transformation. As shown in section 5.3, the $T$-transformation, $\tau_{\mathrm{IR}} \rightarrow \tau_{\mathrm{IR}}+1$, corresponds to

$$
\theta_{\mathrm{IR}} \rightarrow \theta_{\mathrm{IR}}+\frac{\pi}{2}
$$


and therefore maps the monopole of the minimal magnetic charge to a dyon that has half the electric charge of fundamental quark. This implies that the "electric charge" here is not simply the electric charge associated with the $\mathrm{SU}(2)$ vector multiplet in figure 1. Instead, this electric charge is interpreted as a linear combination of the electric charge associated with the $\mathrm{SU}(2)$ vector multiplet sector and those associated with the $\left(A_{1}, D_{4}\right)$ sectors. Indeed, it was shown in $[8]$ that $\operatorname{PSL}(2, \mathbb{Z})$ naturally acts on a modified electro-magnetic charge lattice where the "electric charge" is such a linear combination of charges arising from the three sectors. ${ }^{26}$ This linear combination naturally arises when constructing the $\left(A_{3}, A_{3}\right)$ theory as the IR limit of type IIB string theory on a Calabi-Yau singularity. With this modified charge lattice, the minimal electric charge is now half the charge of fundamental quark, ${ }^{27}$ which is consistent with our T-transformation (6.2).

There are obviously many interesting open problems. We list some of them below.

- While we have focused on the $\left(A_{1}, D_{k}\right)$ theories for even $k$, there are also those theories for odd $k$. Since they also have $\mathrm{SU}(2)$ flavor symmetry that can be gauged, one can consider the generalization of our work to this latter class of theories. One difficulty is that the relevant irregular state in this case cannot be obtained in a colliding limit. Therefore, it is not straightforward to derive the action of the Heisenberg algebra on the irregular state.

- It is also interesting to generalize our work to $\mathrm{SU}(N)$ gauge theories coupled to $\mathrm{AD}$ theories. For that, we need a $\mathrm{U}(N)$-version of the generalized AGT correspondence. A careful study on the $\mathrm{SU}(3)$-version of the generalized AGT correspondence has been carried out in [24].

- It would be interesting to study the Nekrasov-Shatashvili limit [37] of the $\Omega$-deformed $\left(A_{3}, A_{3}\right)$ theory. In this limit, combining the results of [38-41] with our formula, one can evaluate the deformed prepotential of the $\left(A_{3}, A_{3}\right)$ theory including both the perturbative and instanton parts.

- The uplift of the AGT correspondence to five dimensions are known [42-51]. It would be interesting to search for a $5 \mathrm{~d}$ uplift of our results. That would shed some light on the relation between the $4 \mathrm{~d} D_{2 n}(\mathrm{SU}(2))$ theory and the $5 \mathrm{~d} \widehat{D}_{2 n}(\mathrm{SU}(2))$ theory $[7,52]$. More generally, it would be interesting to study how our results are phrased in terms of the $W_{1+\infty}$ algebra and the DIM algebra along the lines of [53-65].

\footnotetext{
${ }^{26}$ The "magnetic charge" here is simply associated with the $\mathrm{SU}(2)$ vector multiplet without mixing with those arising from the $\left(A_{1}, D_{4}\right)$ sectors.

${ }^{27}$ This can be seen from the discussions around eq. (2.64) of [8], where $\operatorname{deg} X$ and $\operatorname{rank} X$ are respectively the (modified) electric and magnetic charges naturally acted on by $\operatorname{PSL}(2, \mathbb{Z})$. Their discussions here apply to a larger class of theories labeled by $p=2,3,4$ and 6 , and the $\left(A_{3}, A_{3}\right)$ theory corresponds to the $p=4$ case. As the authors explain there, $\operatorname{deg} X$ is quantized in the unit of $1 / p=1 / 4$ when it is normalized so that the $\mathrm{W}$-boson has charge 1 . This means that the minimal value of the modified electric charge, $\operatorname{deg} X$, is half the charge of fundamental quark.
} 
- It was shown in [35] that the Schur indices of the $\left(A_{3}, A_{3}\right)$ theory and $\mathrm{SU}(2)$ gauge theory with four flavors are related by a change of variables involving

$$
q \rightarrow q^{2}
$$

where $q$ is now the superconformal fugacity of the index (See also [66] for similar relations to $\mathcal{N}=4$ super Yang-Mills theories). Although this $q$ is different from our $q$ in (6.1), it would be very interesting to study how these two peculiar relations are connected.

- Our discussion in the previous bullet suggests that the Schur index and the prepotential might generally be related. Since the Schur index is identified as the character of the associated chiral algebra [67], this then suggests a possible connection between the chiral algebra and the prepotential. Such a connection has already been suggested in the application of the ODE/IM correspondence to quantum SW-curves [68]. It would be interesting to study this connection further.

- A replacement similar to (6.1) arises when considering the Nekrasov partition functions of $S O / S p$ gauge theories in connection to $S U$ gauge theories [69, 70]. This seems to suggest that a $\mathbb{Z}_{2}$-orbifolding plays some role similarly in our case. It would be interesting to study this possibility further.

\section{Acknowledgments}

We would like to thank Matthew Buican, Katsushi Ito, Kazunobu Maruyoshi, Hiraku Nakajima, Satoshi Nawata, Takuya Okuda, Jaewon Song, Yuji Tachikawa, and Wenbin Yan for various illuminating discussions. We would like to particularly thank Kazunobu Maruyoshi for drawing our attention to the reference [34] when one of us (T.N.) had a discussion with him during the international conference "KEK Theory Workshop 2018." T.N. also thanks KEK theory group for hosting the wonderful workshop with various opportunities of discussions. T.N.'s research is partially supported by JSPS Grant-in-Aid for Early-Career Scientists 18K13547. The work of T. U. is supported by Grant-in-Aid for JSPS Research Fellows 19J11212. 


\section{A Formulae for Nekrasov partition functions}

Here, we list formulae for contributions to the Nekrasov partition function of U(2) gauge theories $[1-5,71]$. As discussed around eq. (1.1), the instanton part of the partition function is written as a sum over pairs of Young diagrams, $\left(Y_{1}, Y_{2}\right)$. For each $\left(Y_{1}, Y_{2}\right)$, the $\mathrm{U}(2)$ vector multiplet contributes the following factor

$$
\mathcal{Z}_{Y_{1}, Y_{2}}^{\mathrm{vec}}(a) \equiv \prod_{i, j=1}^{2} \prod_{s \in Y_{i}} \frac{1}{-E_{Y_{i}, Y_{j}}\left(a_{i}-a_{j}, s\right)+\epsilon_{1}+\epsilon_{2}} \prod_{t \in Y_{j}} \frac{1}{E_{Y_{j}, Y_{i}}\left(a_{j}-a_{i}, t\right)},
$$

where $a_{1}=-a_{2}=a$ corresponds to the Coulomb branch parameter, and

$$
E_{Y_{1}, Y_{2}}(a, s) \equiv a-\epsilon_{1} L_{Y_{2}}(s)+\epsilon_{2}\left(A_{Y_{1}}(s)+1\right) .
$$

Here $L_{Y}(s)$ and $A_{Y}(s)$ are defined as follows. For a Young diagram $Y=\left\{\lambda_{1} \geq \lambda_{2} \geq \cdots\right\}$, we denote its transpose by $\left\{\lambda_{1}^{\prime} \geq \lambda_{2}^{\prime} \geq \cdots\right\}$. We also denote by $s=(i, j)$ a position in a Young diagram $Y$. Then the leg-length and arm-length are defined by $L_{Y}(s) \equiv \lambda_{j}^{\prime}-i$ and $A_{Y}(s) \equiv \lambda_{i}-j$, respectively.

The contribution from a fundamental hypermultiplet of $\mathrm{U}(2)$ is given by

$$
\mathcal{Z}_{Y_{1}, Y_{2}}^{\text {fund }}(a, m) \equiv \prod_{i=1}^{2} \prod_{s \in Y_{i}}\left(\phi\left(a_{i}, s\right)-m+\epsilon_{1}+\epsilon_{2}\right),
$$

where

$$
\phi(a, s) \equiv a+\epsilon_{1}(i-1)+\epsilon_{2}(j-1)
$$

and $m$ is the mass of the hypermultiplet. The contribution from a bi-fundamental hypermultiplet of $\mathrm{U}(2) \times \mathrm{U}(2)$ is written as

$$
\begin{aligned}
\mathcal{Z}_{Y_{1}, Y_{2} ; W_{1}, W_{2}}^{\text {bifund }}(a, b, \alpha) \equiv & \prod_{i, j=1}^{2} \prod_{s \in Y_{i}}\left(E\left(a_{i}-b_{j}, Y_{i}, W_{j}, s\right)-\alpha\right) \\
& \times \prod_{t \in W_{j}}\left(\epsilon_{1}+\epsilon_{2}-E\left(b_{j}-a_{i}, W_{j}, Y_{i}, t\right)-\alpha\right)
\end{aligned}
$$

where $\left(Y_{1}, Y_{2}\right)$ and $\left(W_{1}, W_{2}\right)$ correspond to torus fixed points on the instanton moduli spaces of two $\mathrm{U}(2)$ gauge groups, and $a$ and $b$ are the Coulomb branch parameters of these two $\mathrm{U}(2)$. The extra parameter, $\alpha$, stands for the mass parameter of the bi-fundamental hypermultiplet.

\section{B Orthogonal basis}

Here we list the first few examples of states $\left|a ; Y_{1}, Y_{2}\right\rangle$ in the basis of the highest weight module of $\operatorname{Vir} \oplus H$ that we have used in section 4. These states are defined as solutions to (4.2), and were first found in [34]. For ease of reference, we here denote the highest weight by $P$ instead of $a$, as in [34]. 
To describe these states, we first decompose the space of states by the level of descendants. Here, the "level" is the sum of the levels of the Virasoro part and the Heisenberg part, so that $L_{-k_{1}}^{m_{1}} \cdots L_{-k_{p}}^{m_{p}} a_{-\ell_{1}}^{n_{1}} \cdots a_{-\ell_{q}}^{n_{q}}|P\rangle$ has level $\sum_{i=1}^{p} m_{i} k_{i}+\sum_{j=1}^{q} n_{j} \ell_{j}$. The basis $\left\{\left|P ; Y_{1}, Y_{2}\right\rangle\right\}$ found in [34] is such that $\left|P ; Y_{1}, Y_{2}\right\rangle$ for $\left|Y_{1}\right|+\left|Y_{2}\right|=k$ span the space of level$k$ descendants. Recall here that we are rescaling dimensionful parameters so that $\epsilon_{1} \epsilon_{2}=1$. Therefore we set $\epsilon_{1}=1 / \epsilon_{2}=\mathrm{b}$ when computing the r.h.s. of (4.2). In this case, the Liouville charge is written as $Q=\mathrm{b}+1 / \mathrm{b}$. Below, we list the first few examples of $\left|P ; Y_{1}, Y_{2}\right\rangle$ :

$$
\begin{aligned}
& |P ; \emptyset, \emptyset\rangle=|P\rangle, \\
& |P ; \square, \emptyset\rangle=\left(-i\left(\mathrm{~b}+\mathrm{b}^{-1}+2 P\right) a_{-1}-L_{-1}\right)|P\rangle, \\
& |P ; \square, \emptyset\rangle=\left(-i \mathrm{~b}^{-1}\left(\mathrm{~b}+\mathrm{b}^{-1}+2 P\right)\left(\mathrm{b}+2 \mathrm{~b}^{-1}+2 P\right) a_{-2}\right. \\
& -\left(\mathrm{b}+\mathrm{b}^{-1}+2 P\right)\left(\mathrm{b}+2 \mathrm{~b}^{-1}+2 P\right) a_{-1}^{2} \\
& \left.+2 i\left(\mathrm{~b}+2 \mathrm{~b}^{-1}+2 P\right) a_{-1} L_{-1}-\mathrm{b}^{-1}\left(\mathrm{~b}+\mathrm{b}^{-1}+2 P\right) L_{-2}+L_{-1}^{2}\right)|P\rangle, \\
& |P ; \square, \emptyset\rangle=\left(-i \mathrm{~b}\left(\mathrm{~b}+\mathrm{b}^{-1}+2 P\right)\left(2 \mathrm{~b}+\mathrm{b}^{-1}+2 P\right) a_{-2}\right. \\
& -\left(\mathrm{b}+\mathrm{b}^{-1}+2 P\right)\left(2 \mathrm{~b}+\mathrm{b}^{-1}+2 P\right) a_{-1}^{2} \\
& \left.+2 i\left(2 \mathrm{~b}+\mathrm{b}^{-1}+2 P\right) a_{-1} L_{-1}-\mathrm{b}\left(\mathrm{b}+\mathrm{b}^{-1}+2 P\right) L_{-2}+L_{-1}^{2}\right)|P\rangle, \\
& |P ; \square, \square\rangle=\left(-i\left(\mathrm{~b}+\mathrm{b}^{-1}\right) a_{-2}-\left(\mathrm{b}^{2}+\mathrm{b}^{-2}+1-4 P^{2}\right) a_{-1}^{2}\right. \\
& \left.+2 i\left(\mathrm{~b}+\mathrm{b}^{-1}\right) a_{-1} L_{-1}-L_{-2}+L_{-1}^{2}\right)|P\rangle, \\
& |P ; \boxminus, \emptyset\rangle=\left(-2 i \mathrm{~b}^{-2}\left(\mathrm{~b}+\mathrm{b}^{-1}+2 P\right)\left(\mathrm{b}+2 \mathrm{~b}^{-1}+2 P\right)\left(\mathrm{b}+3 \mathrm{~b}^{-1}+2 P\right) a_{-3}\right. \\
& -3 \mathrm{~b}^{-1}\left(\mathrm{~b}+\mathrm{b}^{-1}+2 P\right)\left(\mathrm{b}+2 \mathrm{~b}^{-1}+2 P\right)\left(\mathrm{b}+3 \mathrm{~b}^{-1}+2 P\right) a_{-2} a_{-1} \\
& +i\left(\mathrm{~b}+\mathrm{b}^{-1}+2 P\right)\left(\mathrm{b}+2 \mathrm{~b}^{-1}+2 P\right)\left(\mathrm{b}+3 \mathrm{~b}^{-1}+2 P\right) a_{-1}^{3} \\
& +3 i \mathrm{~b}^{-1}\left(\mathrm{~b}+2 \mathrm{~b}^{-1}+2 P\right)\left(\mathrm{b}+3 \mathrm{~b}^{-1}+2 P\right) a_{-2} L_{-1} \\
& +3\left(\mathrm{~b}+2 \mathrm{~b}^{-1}+2 P\right)\left(\mathrm{b}+3 \mathrm{~b}^{-1}+2 P\right) a_{-1}^{2} L_{-1} \\
& +3 i \mathrm{~b}^{-1}\left(\mathrm{~b}+\mathrm{b}^{-1}+2 P\right)\left(\mathrm{b}+3 \mathrm{~b}^{-1}+2 P\right) a_{-1} L_{-2} \\
& -3 i\left(\mathrm{~b}+3 \mathrm{~b}^{-1}+2 P\right) a_{-1} L_{-1}^{2}-\mathrm{b}^{-2}\left(\mathrm{~b}+4 \mathrm{~b}^{-1}+4 P\right)\left(\mathrm{b}+\mathrm{b}^{-1}+2 P\right) L_{-3} \\
& \left.+\mathrm{b}^{-1}\left(3 \mathrm{~b}+5 \mathrm{~b}^{-1}+6 P\right) L_{-2} L_{-1}-L_{-1}^{3}\right)|P\rangle, \\
& |P ; \square, \emptyset\rangle=\left(-i\left(\mathrm{~b}+\mathrm{b}^{-1}+2 P\right)\left(\mathrm{b}+2 \mathrm{~b}^{-1}+2 P\right)\left(2 \mathrm{~b}+\mathrm{b}^{-1}+2 P\right) a_{-3}\right. \\
& -\left(\mathrm{b}+\mathrm{b}^{-1}\right)\left(\mathrm{b}+\mathrm{b}^{-1}+2 P\right)\left(\mathrm{b}+2 \mathrm{~b}^{-1}+2 P\right)\left(2 \mathrm{~b}+\mathrm{b}^{-1}+2 P\right) a_{-2} a_{-1} \\
& +i\left(\mathrm{~b}+\mathrm{b}^{-1}+2 P\right)\left(\mathrm{b}+2 \mathrm{~b}^{-1}+2 P\right)\left(2 \mathrm{~b}+\mathrm{b}^{-1}+2 P\right) a_{-1}^{3} \\
& +i\left(\mathrm{~b}+\mathrm{b}^{-1}\right)\left(\mathrm{b}+2 \mathrm{~b}^{-1}+2 P\right)\left(2 \mathrm{~b}+\mathrm{b}^{-1}+2 P\right) a_{-2} L_{-1} \\
& +3\left(\mathrm{~b}+2 \mathrm{~b}^{-1}+2 P\right)\left(2 \mathrm{~b}+\mathrm{b}^{-1}+2 P\right) a_{-1}^{2} L_{-1} \\
& +i\left(\mathrm{~b}+\mathrm{b}^{-1}+2 P\right)\left(\mathrm{b}^{2}+5+\mathrm{b}^{-2}+2\left(\mathrm{~b}+\mathrm{b}^{-1}\right) P\right) a_{-1} L_{-2} \\
& -i\left(5 \mathrm{~b}+5 \mathrm{~b}^{-1}+6 P\right) a_{-1} L_{-1}^{2}-\left(\mathrm{b}+\mathrm{b}^{-1}+2 P\right)^{2} L_{-3} \\
& \left.+\left(\mathrm{b}^{2}+3+\mathrm{b}^{-2}+2\left(\mathrm{~b}+\mathrm{b}^{-1}\right) P\right) L_{-2} L_{-1}-L_{-1}^{3}\right)|P\rangle,
\end{aligned}
$$




$$
\begin{aligned}
&|P ; \square \square, \emptyset\rangle=(-2 i \mathrm{~b}^{2}\left(\mathrm{~b}+\mathrm{b}^{-1}+2 P\right)\left(3 \mathrm{~b}+\mathrm{b}^{-1}+2 P\right)\left(2 \mathrm{~b}+\mathrm{b}^{-1}+2 P\right) a_{-3} \\
&-3 \mathrm{~b}\left(\mathrm{~b}+\mathrm{b}^{-1}+2 P\right)\left(3 \mathrm{~b}+\mathrm{b}^{-1}+2 P\right)\left(2 \mathrm{~b}+\mathrm{b}^{-1}+2 P\right) a_{-2} a_{-1} \\
&+i\left(\mathrm{~b}+\mathrm{b}^{-1}+2 P\right)\left(3 \mathrm{~b}+\mathrm{b}^{-1}+2 P\right)\left(2 \mathrm{~b}+\mathrm{b}^{-1}+2 P\right) a_{-1}^{3} \\
&+3 i \mathrm{~b}\left(3 \mathrm{~b}+\mathrm{b}^{-1}+2 P\right)\left(2 \mathrm{~b}+\mathrm{b}^{-1}+2 P\right) a_{-2} L_{-1} \\
&+3\left(3 \mathrm{~b}+\mathrm{b}^{-1}+2 P\right)\left(2 \mathrm{~b}+\mathrm{b}^{-1}+2 P\right) a_{-1}^{2} L_{-1} \\
&+3 i \mathrm{~b}\left(\mathrm{~b}+\mathrm{b}^{-1}+2 P\right)\left(3 \mathrm{~b}+\mathrm{b}^{-1}+2 P\right) a_{-1} L_{-2} \\
&-3 i\left(3 \mathrm{~b}+\mathrm{b}^{-1}+2 P\right) a_{-1} L_{-1}^{2}-\mathrm{b}^{2}\left(\mathrm{~b}+\mathrm{b}^{-1}+2 P\right)\left(4 \mathrm{~b}+\mathrm{b}^{-1}+4 P\right) L_{-3} \\
&\left.+\mathrm{b}\left(5 \mathrm{~b}+3 \mathrm{~b}^{-1}+6 P\right) L_{-2} L_{-1}-L_{-1}^{3}\right)|P\rangle \\
& \mid-2 i \mathrm{~b}^{-1}\left(\mathrm{~b}+\mathrm{b}^{-1}\right)\left(\mathrm{b}+\mathrm{b}^{-1}+2 P\right) a_{-3} \\
&-\mathrm{b}^{-1}\left(\mathrm{~b}+\mathrm{b}^{-1}+2 P\right)\left(3 \mathrm{~b}^{2}+3+2 \mathrm{~b}^{-2}-2 \mathrm{~b}^{-1} P-4 P^{2}\right) a_{-2} a_{-1} \\
&+i\left(\mathrm{~b}+\mathrm{b}^{-1}+2 P\right)\left(\mathrm{b}^{2}+1+2 \mathrm{~b}^{-2}-2 \mathrm{~b}^{-1} P-4 P^{2}\right) a_{-1}^{3} \\
&+i \mathrm{~b}^{-1}\left(3 \mathrm{~b}^{2}+7+2 \mathrm{~b}^{-2}+\left(4 \mathrm{~b}+6 \mathrm{~b}^{-1}\right) P+4 P^{2}\right) a_{-2} L_{-1} \\
&+\left(3 \mathrm{~b}^{2}+7+6 \mathrm{~b}^{-2}+\left(4 \mathrm{~b}+2 \mathrm{~b}^{-1}\right) P-4 P^{2}\right) a_{-1}^{2} L_{-1} \\
&+i \mathrm{~b}^{-1}\left(3 \mathrm{~b}+\mathrm{b}^{-1}-2 P\right)\left(\mathrm{b}+\mathrm{b}^{-1}+2 P\right) a_{-1} L_{-2} \\
&-i\left(3 \mathrm{~b}+5 \mathrm{~b}^{-1}+2 P\right) a_{-1} L_{-1}^{2}-\mathrm{b}^{-1}\left(\mathrm{~b}+\mathrm{b}^{-1}+2 P\right) L_{-3} \\
&\left.+\mathrm{b}^{-1}\left(3 \mathrm{~b}+\mathrm{b}^{-1}+2 P\right) L_{-2} L_{-1}-L_{-1}^{3}\right)|P\rangle \\
&\square\rangle=( 2 i \mathrm{~b}\left(\mathrm{~b}+\mathrm{b}^{-1}\right)\left(\mathrm{b}+\mathrm{b}^{-1}+2 P\right) a_{-3} \\
&-\mathrm{b}\left(\mathrm{b}+\mathrm{b}^{-1}+2 P\right)\left(2 \mathrm{~b}^{2}+3+3 \mathrm{~b}^{-2}-2 \mathrm{~b} P-4 P^{2}\right) a_{-2} a_{-1} \\
&+i\left(\mathrm{~b}+\mathrm{b}^{-1}+2 P\right)\left(2 \mathrm{~b}^{2}+1+\mathrm{b}^{-2}-2 \mathrm{~b} P-4 P^{2}\right) a_{-1}^{3} \\
&+i \mathrm{~b}\left(2 \mathrm{~b}^{2}+7+3 \mathrm{~b}^{-2}+\left(6 \mathrm{~b}+4 \mathrm{~b}^{-1}\right) P+4 P^{2}\right) a_{-2} L_{-1} \\
&+\left(6 \mathrm{~b}^{2}+7+3 \mathrm{~b}^{-2}+\left(2 \mathrm{~b}+4 \mathrm{~b}^{-1}\right) P-4 P^{2}\right) a_{-1}^{2} L_{-1} \\
&+i \mathrm{~b}\left(\mathrm{~b}+3 \mathrm{~b}^{-1}-2 P\right)\left(\mathrm{b}+\mathrm{b}^{-1}+2 P\right) a_{-1} L_{-2} \\
&-i\left(5 \mathrm{~b}+3 \mathrm{~b}^{-1}+2 P\right) a_{-1} L_{-1}^{2}-\mathrm{b}\left(\mathrm{b}+\mathrm{b}^{-1}+2 P\right) L_{-3} \\
&\left.+\mathrm{b}\left(\mathrm{b}+3 \mathrm{~b}^{-1}+2 P\right) L_{-2} L_{-1}-L_{-1}^{3}\right)|P\rangle \\
&|P ; \square, \square\rangle=(\mathrm{B} .9 \\
& \square=(\mathrm{B}
\end{aligned}
$$

Note that there are also states obtained by exchanging two Young diagrams in each of the above, whose expressions are simply obtained via the relation $\left|P ; Y_{2}, Y_{1}\right\rangle=\left|-P ; Y_{1}, Y_{2}\right\rangle$.

\section{Prepotential with massive deformations}

As discussed in section 5.4, we have checked that the instanton part of the prepotential of the $\left(A_{3}, A_{3}\right)$ theory is invariant under (5.45), up to terms affected by the $\mathrm{U}(1)$-factor. Indeed, our computation shows that

$$
\mathcal{F}_{\text {inst }}^{\left(A_{3}, A_{3}\right)}=\sum_{k=-1}^{\infty} \mathcal{F}_{2 k} a^{-2 k},
$$


where

$$
\begin{gathered}
\mathcal{F}_{-2}=q^{2}+\frac{13}{8} q^{4}+\frac{23}{6} q^{6}+\frac{2701}{256} q^{8}+\mathcal{O}\left(q^{10}\right), \\
\mathcal{F}_{2}=\frac{q}{2} M u_{1} u_{2}+\frac{q^{2}}{16}\left(-4 d_{1}^{2} d_{2}^{2} M^{2}-8 d_{1}^{2} M^{2} m_{2}-d_{1}^{2} u_{2}^{2}\right. \\
\left.\quad-8 d_{2}^{2} M^{2} m_{1}-d_{2}^{2} u_{1}^{2}-16 M^{2} m_{1} m_{2}-2 m_{1} u_{2}^{2}-2 m_{2} u_{1}^{2}\right) \\
+\frac{q^{3}}{24}\left(12 d_{1}^{3} d_{2} M^{2}-6 d 1^{2} d_{2} M u_{1}+12 d_{1} d_{2}^{3} M^{2}-6 d_{1} d_{2}^{2} M u_{2}+16 d_{1} d_{2} M^{3}\right. \\
+24 d_{1} d_{2} M^{2} m_{1}+24 d_{1} d_{2} M^{2} m_{2}+3 d 1 d_{2} u_{1}^{2}+3 d_{1} d_{2} u_{2}^{2} \\
\left.\quad-12 d 1 M m_{2} u_{2}-12 d_{2} M m_{1} u_{1}+12 M u_{1} u_{2}\right)+\mathcal{O}\left(q^{4}\right), \\
\mathcal{F}_{4}=\frac{3 q^{2}}{64}\left(4 d_{1}^{2} M^{2} u_{2}^{2}+4 d_{2}^{2} M^{2} u_{1}^{2}+8 M^{2} m_{1} u_{2}^{2}+8 M^{2} m_{2} u_{1}^{2}+u_{1}^{2} u_{2}^{2}\right) \\
+\frac{q^{3}}{48}\left(-12 d_{1}^{2} d_{2}^{2} M u_{1} u_{2}+20 d_{1}^{2} d_{2} M^{3} u_{1}-24 d_{1}^{2} M m_{2} u_{1} u_{2}+20 d_{1} d_{2}^{2} M^{3} u_{2}\right. \\
\quad-18 d_{1} d_{2} M^{2} u_{1}^{2}-18 d_{1} d_{2} M^{2} u_{2}^{2}+40 d_{1} M^{3} m_{2} u_{2}+5 d_{1} M u_{2}^{3}-24 d_{2}^{2} M m_{1} u_{1} u_{2} \\
\left.\quad+40 d_{2} M^{3} m_{1} u_{1}+5 d_{2} M u_{1}^{3}-48 M m_{1} m_{2} u_{1} u_{2}\right)+\mathcal{O}\left(q^{4}\right) .
\end{gathered}
$$

One can explicitly check that these expressions are all invariant under (5.45). ${ }^{28}$

As mentioned at the end of section 5.4, imposing the invariance under (5.45) constrains the possible form of $\mathcal{F}_{k>0}$ in (5.43). In particular, when combined with the trivial symmetry of the theory under exchanging two $\left(A_{1}, D_{4}\right)$ sectors, it determines $\mathcal{F}_{k}$ up to undetermined $T$-invariant functions. In the rest of this appendix, we explicitly show this for $\mathcal{F}_{2}$.

To that end, it is useful for us below to re-express (5.45) in terms of the IR gauge coupling $\tau_{\mathrm{IR}}$ instead of the UV gauge coupling $q$, i.e.,

$$
\tau_{\mathrm{IR}} \rightarrow \tau_{\mathrm{IR}}+1, \quad d_{1} \rightarrow \frac{\theta_{3}^{2}}{\theta_{4}^{2}} d_{1}-\frac{\theta_{2}^{2}}{\theta_{4}^{2}} d_{2}, \quad d_{2} \rightarrow i d_{2}, \quad m_{2} \rightarrow-m_{2}, \quad u_{2} \rightarrow i u_{2},
$$

where we used the shorthands $\theta_{2} \equiv \theta_{2}\left(q_{\mathrm{IR}}^{2}\right), \theta_{3} \equiv \theta_{3}\left(q_{\mathrm{IR}}^{2}\right)$ and $\theta_{4} \equiv \theta_{4}\left(q_{\mathrm{IR}}^{2}\right)$. Note that $\tau_{\mathrm{IR}} \rightarrow \tau_{\mathrm{IR}}+1$ implies $\theta_{3}\left(q_{\mathrm{IR}}^{2}\right) \leftrightarrow \theta_{4}\left(q_{\mathrm{IR}}^{2}\right)$ and $\theta_{2}\left(q_{\mathrm{IR}}^{2}\right) \rightarrow e^{\frac{\pi i}{4}} \theta_{2}\left(q_{\mathrm{IR}}^{2}\right)$.

The $\left(A_{3}, A_{3}\right)$ theory also has a trivial symmetry under the permutation of two $\left(A_{1}, D_{4}\right)$ theories in the quiver description in figure 1 . This permutation symmetry implies that $\mathcal{F}_{k>0}$ must also be invariant under

$$
d_{1} \longleftrightarrow d_{2}, \quad m_{1} \longleftrightarrow m_{2}, \quad u_{1} \longleftrightarrow u_{2}
$$

Below, we show that demanding the invariance of $\mathcal{F}_{k>0}$ under (C.5) and (C.6) strongly constrains the possible form of $\mathcal{F}_{k>0}$, especially focusing on $\mathcal{F}_{2}$.

For that, first note that $\mathcal{F}_{2}$ is a meromorphic function of $d_{i}, m_{i}, u_{i}, M$ and $\tau_{\mathrm{IR}}$ such that $\mathcal{F}_{2}=0$ when $d_{i}=m_{i}=u_{i}=M=0$. Moreover, since the prepotential is of dimension two, $\mathcal{F}_{2}$ is of dimension four. These mean that $\mathcal{F}_{2}$ is a polynomial of $d_{i}, m_{i}, u_{i}$ and $M$ whose coefficients depend on $\tau_{\mathrm{IR}}$. The invariance under (C.5) and (C.6) implies that these

\footnotetext{
${ }^{28}$ Note that $\mathcal{F}_{0}$ depends on the $\mathrm{U}(1)$-factor that we have not identified.
} 
coefficients are correlated in a highly non-trivial way. For example, let us consider the possible term

$$
g\left(q_{\mathrm{IR}}\right) m_{1} u_{1} u_{2}
$$

where $g\left(q_{\mathrm{IR}}\right)$ is an unknown function of $q_{\mathrm{IR}}$. Since it is of dimension four, this term might appear in $\mathcal{F}_{2}$. The constraint (C.6) then implies that (C.7) must be accompanied with $g\left(q_{\mathrm{IR}}\right) m_{2} u_{1} u_{2}$. Therefore, (C.7) can appear in $\mathcal{F}_{2}$ only as a part of

$$
g\left(q_{\mathrm{IR}}\right)\left(m_{1}+m_{2}\right) u_{1} u_{2} .
$$

However, the $T$-invariance (C.5) now requires

$$
i g\left(\widetilde{q}_{\mathrm{IR}}\right)\left(m_{1}-m_{2}\right) u_{1} u_{2}=g\left(q_{\mathrm{IR}}\right)\left(m_{1}+m_{2}\right) u_{1} u_{2},
$$

where $\widetilde{q}_{\mathrm{IR}}$ is the image of $q_{\mathrm{IR}}$ under the $T$-transformation. For this equality to hold as a function of $q_{\mathrm{IR}}, m_{i}$ and $u_{i}$, we need $g\left(q_{\mathrm{IR}}\right)=0$. Hence, the invariance under the $T$ transformation together with the permutation symmetry (C.6) prohibits the term proportional to $m_{1} u_{1} u_{2}$ or $m_{2} u_{1} u_{2}$ in $\mathcal{F}_{2}$.

The invariance under (C.5) and (C.6) also constrains the coefficients of non-vanishing terms in $\mathcal{F}_{2}$. Indeed, we find that the most general expression for $\mathcal{F}_{2}$ that is invariant under (C.5) and (C.6) is written in terms of only 54 coefficients $h_{1}\left(q_{\mathrm{Ir}}\right), \cdots, h_{54}\left(q_{\mathrm{IR}}\right)$ as follows.

$$
\begin{aligned}
& \mathcal{F}_{2}\left(q, M,\left\{m_{i}\right\},\left\{u_{i}\right\},\left\{d_{i}\right\}\right) \\
& =h_{1}\left(q_{\mathrm{IR}}\right) M^{4}+h_{2}\left(q_{\mathrm{IR}}\right) M^{2}\left(m_{1}^{2}+m_{2}^{2}\right)+h_{3}\left(q_{\mathrm{IR}}\right) M^{2} m_{1} m_{2} \frac{\theta_{2}^{4}}{\theta_{3}^{2} \theta_{4}^{2}}+h_{4}\left(q_{\mathrm{IR}}\right)\left(m_{1}^{4}+m_{2}^{4}\right) \\
& +h_{5}\left(q_{\mathrm{IR}}\right) m_{1}^{2} m_{2}^{2}+h_{6}\left(q_{\mathrm{IR}}\right) m_{1} m_{2}\left(m_{1}^{2}+m_{2}^{2}\right) \frac{\theta_{2}^{4}}{\theta_{3}^{2} \theta_{4}^{2}} \\
& +h_{7}\left(q_{\mathrm{IR}}\right) M u_{1} u_{2} \frac{\theta_{2}^{2}}{\theta_{3} \theta_{4}}+h_{8}\left(q_{\mathrm{IR}}\right)\left(m_{1} u_{1}^{2}+m_{2} u_{2}^{2}\right)+h_{9}\left(q_{\mathrm{IR}}\right)\left(m_{2} u_{1}^{2}+m_{1} u_{2}^{2}\right) \frac{\theta_{2}^{4}}{\theta_{3}^{2} \theta_{4}^{2}} \\
& +h_{10}\left(q_{\mathrm{IR}}\right) M^{2} B_{2}\left(u_{i}, d_{i}\right)+h_{11}\left(q_{\mathrm{IR}}\right) m_{1} m_{2} B_{2}\left(u_{i}, d_{i}\right) \frac{\theta_{2}^{4}}{\theta_{3}^{2} \theta_{4}^{2}}+h_{12}\left(q_{\mathrm{IR}}\right) M B_{3}^{(1)}\left(m_{i}, u_{i}, d_{i}\right) \\
& +h_{13}\left(q_{\mathrm{IR}}\right) M B_{3}^{(2)}\left(m_{i}, u_{i}, d_{i}\right)+h_{14}\left(q_{\mathrm{IR}}\right) B_{4}^{(1)}\left(m_{i}, u_{i}, d_{i}\right)+h_{15}\left(q_{\mathrm{IR}}\right) B_{4}^{(2)}\left(m_{i}, u_{i}, d_{i}\right) \\
& +h_{16}\left(q_{\mathrm{IR}}\right) M^{3} D_{1}\left(d_{i}\right)+h_{17}\left(q_{\mathrm{IR}}\right) M\left(m_{1}^{2}+m_{2}^{2}\right) D_{1}\left(d_{i}\right)+h_{18}\left(q_{\mathrm{IR}}\right) M m_{1} m_{2} D_{1}\left(d_{i}\right) \frac{\theta_{2}^{4}}{\theta_{3}^{2} \theta_{4}^{2}} \\
& +h_{19}\left(q_{\mathrm{IR}}\right) M^{2} E_{2}^{(1)}\left(m_{i}, d_{i}\right)+h_{20}\left(q_{\mathrm{IR}}\right) M^{2} E_{2}^{(2)}\left(m_{i}, d_{i}\right)+h_{21}\left(q_{\mathrm{IR}}\right) m_{1} m_{2} E_{2}^{(1)}\left(m_{i}, d_{i}\right) \frac{\theta_{2}^{4}}{\theta_{3}^{2} \theta_{4}^{2}} \\
& +h_{22}\left(q_{\mathrm{IR}}\right) m_{1} m_{2} E_{2}^{(2)}\left(m_{i}, d_{i}\right) \frac{\theta_{2}^{4}}{\theta_{3}^{2} \theta_{4}^{2}}+h_{23}\left(q_{\mathrm{IR}}\right) E_{4}^{(1)}\left(m_{i}, d_{i}\right)+h_{24}\left(q_{\mathrm{IR}}\right) E_{4}^{(2)}\left(m_{i}, d_{i}\right) \\
& +h_{25}\left(q_{\mathrm{IR}}\right) u_{1} u_{2} D_{1}\left(d_{i}\right) \frac{\theta_{2}^{2}}{\theta_{3} \theta_{4}}+h_{26}\left(q_{\mathrm{IR}}\right) F_{4}^{(1)}\left(u_{i}, d_{i}\right)+h_{27}\left(q_{\mathrm{IR}}\right) F_{4}^{(2)}\left(u_{i}, d_{i}\right) \\
& +h_{28}\left(q_{\mathrm{IR}}\right) M G_{3}^{(1)}\left(u_{i}, d_{i}\right)+h_{29}\left(q_{\mathrm{IR}}\right) M G_{3}^{(2)}\left(u_{i}, d_{i}\right)+h_{30}\left(q_{\mathrm{IR}}\right) G_{4}^{(1)}\left(m_{i}, u_{i}, d_{i}\right) \\
& +h_{31}\left(q_{\mathrm{IR}}\right) G_{4}^{(2)}\left(m_{i}, u_{i}, d_{i}\right)+h_{32}\left(q_{\mathrm{IR}}\right) G_{4}^{(3)}\left(m_{i}, u_{i}, d_{i}\right)+h_{33}\left(q_{\mathrm{IR}}\right) G_{4}^{(4)}\left(m_{i}, u_{i}, d_{i}\right)
\end{aligned}
$$




$$
\begin{aligned}
+ & h_{34}\left(q_{\mathrm{IR}}\right) M^{2}\left(D_{1}\left(d_{i}\right)\right)^{2}+h_{35}\left(q_{\mathrm{IR}}\right) m_{1} m_{2}\left(D_{1}\left(d_{i}\right)\right)^{2} \frac{\theta_{2}^{4}}{\theta_{3}^{2} \theta_{4}^{2}}+h_{36}\left(q_{\mathrm{IR}}\right) M^{2} D_{2}\left(d_{i}\right) \\
& +h_{37}\left(q_{\mathrm{IR}}\right) m_{1} m_{2} D_{2}\left(d_{i}\right) \frac{\theta_{2}^{4}}{\theta_{3}^{2} \theta_{4}^{2}}+h_{38}\left(q_{\mathrm{IR}}\right)\left(m_{1}^{2}+m_{2}^{2}\right) D_{2}\left(d_{i}\right)+h_{39}\left(q_{\mathrm{IR}}\right) M H_{3}^{(1)}\left(m_{i}, d_{i}\right) \\
& +h_{40}\left(q_{\mathrm{IR}}\right) M H_{3}^{(2)}\left(m_{i}, d_{i}\right)+h_{41}\left(q_{\mathrm{IR}}\right) H_{4}^{(1)}\left(m_{i}, d_{i}\right)+h_{42}\left(q_{\mathrm{IR}}\right) H_{4}^{(2)}\left(m_{i}, d_{i}\right) \\
+ & h_{43}\left(q_{\mathrm{IR}}\right) I_{4}^{(1)}\left(u_{i}, d_{i}\right)+h_{44}\left(q_{\mathrm{IR}}\right) I_{4}^{(2)}\left(u_{i}, d_{i}\right)+h_{45}\left(q_{\mathrm{IR}}\right) I_{4}^{(3)}\left(u_{i}, d_{i}\right) \\
+ & h_{46}\left(q_{\mathrm{IR}}\right) M\left(D_{1}\left(d_{i}\right)\right)^{3}+h_{47}\left(q_{\mathrm{IR}}\right) M D_{1}\left(d_{i}\right) D_{2}\left(d_{i}\right)+h_{48}\left(q_{\mathrm{IR}}\right) J_{4}^{(1)}\left(m_{i}, d_{i}\right) \\
& +h_{49}\left(q_{\mathrm{IR}}\right) J_{4}^{(2)}\left(m_{i}, d_{i}\right)+h_{50}\left(q_{\mathrm{IR}}\right) J_{4}^{(3)}\left(m_{i}, d_{i}\right)+h_{51}\left(q_{\mathrm{IR}}\right) J_{4}^{(4)}\left(m_{i}, d_{i}\right) \\
+ & h_{52}\left(q_{\mathrm{IR}}\right)\left(D_{1}\left(d_{i}\right)\right)^{4}+h_{53}\left(q_{\mathrm{IR}}\right)\left(D_{1}\left(d_{i}\right)\right)^{2} D_{2}\left(d_{i}\right)+h_{54}\left(q_{\mathrm{IR}}\right)\left(D_{2}\left(d_{i}\right)\right)^{2}
\end{aligned}
$$

with the following building blocks

$$
\begin{aligned}
& B_{2}\left(u_{i}, d_{i}\right)=\left(\left(u_{1} d_{1}+u_{2} d_{2}\right)+\left(u_{2} d_{1}+u_{1} d_{2}\right) \frac{\theta_{3}^{2}-\theta_{4}^{2}}{\theta_{2}^{2}}\right) \frac{\theta_{3}}{\theta_{4}}, \\
& B_{3}^{(1)}\left(m_{i}, u_{i}, d_{i}\right)=\left(\left(m_{1} u_{1} d_{1}+m_{2} u_{2} d_{2}\right)+\left(m_{2} u_{2} d_{1}+m_{1} u_{1} d_{2}\right) \frac{\theta_{3}^{2}+\theta_{4}^{2}}{\theta_{2}^{2}}\right) \frac{\theta_{3}}{\theta_{4}}, \\
& B_{3}^{(2)}\left(m_{i}, u_{i}, d_{i}\right)=\left(\left(m_{2} u_{1} d_{1}+m_{1} u_{2} d_{2}\right)+\left(m_{1} u_{2} d_{1}+m_{2} u_{1} d_{2}\right) \frac{\theta_{3}^{2}+\theta_{4}^{2}}{\theta_{2}^{2}}\right) \frac{\theta_{2}^{4}}{\theta_{3} \theta_{4}^{3}}, \\
& B_{4}^{(1)}\left(m_{i}, u_{i}, d_{i}\right)=\left(\left(m_{1}^{2} u_{1} d_{1}+m_{2}^{2} u_{2} d_{2}\right)+\left(m_{2}^{2} u_{2} d_{1}+m_{1}^{2} u_{1} d_{2}\right) \frac{\theta_{3}^{2}-\theta_{4}^{2}}{\theta_{2}^{2}}\right) \frac{\theta_{3}}{\theta_{4}}, \\
& B_{4}^{(2)}\left(m_{i}, u_{i}, d_{i}\right)=\left(\left(m_{2}^{2} u_{1} d_{1}+m_{1}^{2} u_{2} d_{2}\right)+\left(m_{1}^{2} u_{2} d_{1}+m_{2}^{2} u_{1} d_{2}\right) \frac{\theta_{3}^{2}-\theta_{4}^{2}}{\theta_{2}^{2}}\right) \frac{\theta_{3}}{\theta_{4}}, \\
& D_{1}\left(d_{i}\right)=\left(\left(d_{1}^{2}+d_{2}^{2}\right)+2 d_{1} d_{2} \frac{\theta_{3}^{2}}{\theta_{2}^{2}}\right) \frac{\theta_{3}^{2}}{\theta_{4}^{2}}, \\
& D_{2}\left(d_{i}\right)=\left(\left(d_{1}^{3} d_{2}+d_{1} d_{2}^{3}\right)+d_{1}^{2} d_{2}^{2} \frac{\theta_{3}^{4}+\theta_{2}^{4}}{\theta_{3}^{2} \theta_{2}^{2}}\right) \frac{\theta_{3}^{4}}{\theta_{4}^{2} \theta_{2}^{2}}, \\
& E_{2}^{(1)}\left(m_{i}, d_{i}\right)=\left(\left(m_{1} d_{1}^{2}+m_{2} d_{2}^{2}\right)+\left(m_{1}+m_{2}\right) d_{1} d_{2} \frac{\theta_{2}^{2}}{\theta_{3}^{2}}\right) \frac{\theta_{3}^{2}}{\theta_{4}^{2}}, \\
& E_{2}^{(2)}\left(m_{i}, d_{i}\right)=\left(\left(m_{2} d_{1}^{2}+m_{1} d_{2}^{2}\right)+\left(m_{1}+m_{2}\right) d_{1} d_{2} \frac{\theta_{2}^{2}}{\theta_{3}^{2}}\right) \frac{\theta_{2}^{4}}{\theta_{4}^{4}}, \\
& E_{4}^{(1)}\left(m_{i}, d_{i}\right)=\left(\left(m_{1}^{3} d_{1}^{2}+m_{2}^{3} d_{2}^{2}\right)+\left(m_{1}^{3}+m_{2}^{3}\right) d_{1} d_{2} \frac{\theta_{2}^{2}}{\theta_{3}^{2}}\right) \frac{\theta_{3}^{2}}{\theta_{4}^{2}}, \\
& E_{4}^{(2)}\left(m_{i}, d_{i}\right)=\left(\left(m_{2}^{3} d_{1}^{2}+m_{1}^{3} d_{2}^{2}\right)+\left(m_{1}^{3}+m_{2}^{3}\right) d_{1} d_{2} \frac{\theta_{2}^{2}}{\theta_{3}^{2}}\right) \frac{\theta_{2}^{4}}{\theta_{4}^{4}}, \\
& F_{4}^{(1)}\left(u_{i}, d_{i}\right)=\left(\left(u_{1}^{2} d_{1}^{2}+u_{2}^{2} d_{2}^{2}\right)+d_{1} d_{2}\left(u_{1}^{2}+u_{2}^{2}\right) \frac{\theta_{2}^{2}}{\theta_{3}^{2}}\right) \frac{\theta_{3}^{2}}{\theta_{4}^{2}}, \\
& F_{4}^{(2)}\left(u_{i}, d_{i}\right)=\left(\left(u_{2}^{2} d_{1}^{2}+u_{1}^{2} d_{2}^{2}\right)+d_{1} d_{2}\left(u_{1}^{2}+u_{2}^{2}\right) \frac{\theta_{2}^{2}}{\theta_{3}^{2}}\right) \frac{\theta_{2}^{4}}{\theta_{4}^{4}}, \\
& G_{3}^{(1)}\left(u_{i}, d_{i}\right)=\left(\left(u_{1} d_{1}^{3}+u_{2} d_{2}^{3}\right)+d_{1} d_{2}\left(\left(u_{1} d_{1}+u_{2} d_{2}\right) \frac{\theta_{3}^{4}+\theta_{2}^{4}+\theta_{3}^{2} \theta_{4}^{2}}{\theta_{3}^{2} \theta_{2}^{2}}+\left(u_{2} d_{1}+u_{1} d_{2}\right) \frac{\theta_{3}^{2}+\theta_{4}^{2}}{\theta_{3}^{2}}\right)\right) \frac{\theta_{3}^{3}}{\theta_{4}^{3}}, \\
& G_{3}^{(2)}\left(u_{i}, d_{i}\right)=\left(\left(u_{2} d_{1}^{3}+u_{1} d_{2}^{3}\right)+d_{1} d_{2}\left(\left(u_{1} d_{1}+u_{2} d_{2}\right) \frac{\theta_{3}^{2}-\theta_{4}^{2}}{\theta_{3}^{2}}+\left(u_{2} d_{1}+u_{1} d_{2}\right) \frac{\theta_{3}^{4}+\theta_{2}^{4}-\theta_{3}^{2} \theta_{4}^{2}}{\theta_{3}^{2} \theta_{2}^{2}}\right)\right) \frac{\theta_{\theta}^{2} \theta_{2}^{2}}{\theta_{4}^{4}}, \\
& G_{4}^{(1)}\left(m_{i}, u_{i}, d_{i}\right)=\left(\left(m_{1} u_{1} d_{1}^{3}+m_{2} u_{2} d_{2}^{3}\right)\right. \\
& \left.+d_{1} d_{2}\left(\left(m_{1} u_{1} d_{1}+m_{2} u_{2} d_{2}\right) \frac{\theta_{3}^{4}+\theta_{2}^{4}-\theta_{3}^{2} \theta_{4}^{2}}{\theta_{3}^{2} \theta_{2}^{2}}+\left(m_{2} u_{2} d_{1}+m_{1} u_{1} d_{2}\right) \frac{\theta_{3}^{2}-\theta_{4}^{2}}{\theta_{3}^{2}}\right)\right) \frac{\theta_{3}^{3}}{\theta_{4}^{3}},
\end{aligned}
$$




$$
\begin{aligned}
& G_{4}^{(2)}\left(m_{i}, u_{i}, d_{i}\right)=\left(\left(m_{2} u_{1} d_{1}^{3}+m_{1} u_{2} d_{2}^{3}\right)\right. \\
& \left.+d_{1} d_{2}\left(\left(m_{2} u_{1} d_{1}+m_{1} u_{2} d_{2}\right) \frac{\theta_{3}^{4}+\theta_{2}^{4}-\theta_{3}^{2} \theta_{4}^{2}}{\theta_{3}^{2} \theta_{2}^{2}}+\left(m_{1} u_{2} d_{1}+m_{2} u_{1} d_{2}\right) \frac{\theta_{3}^{2}-\theta_{4}^{2}}{\theta_{3}^{2}}\right)\right) \frac{\theta_{3} \theta_{2}^{4}}{\theta_{4}^{5}}, \\
& G_{4}^{(3)}\left(m_{i}, u_{i}, d_{i}\right)=\left(\left(m_{1} u_{2} d_{1}^{3}+m_{2} u_{1} d_{2}^{3}\right)\right. \\
& \left.+d_{1} d_{2}\left(\left(m_{2} u_{1} d_{1}+m_{1} u_{2} d_{2}\right) \frac{\theta_{3}^{2}+\theta_{4}^{2}}{\theta_{3}^{2}}+\left(m_{1} u_{2} d_{1}+m_{2} u_{1} d_{2}\right) \frac{\theta_{3}^{4}+\theta_{2}^{4}+\theta_{3}^{2} \theta_{4}^{2}}{\theta_{3}^{2} \theta_{2}^{2}}\right)\right) \frac{\theta_{3}^{2} \theta_{2}^{2}}{\theta_{4}^{4}}, \\
& G_{4}^{(4)}\left(m_{i}, u_{i}, d_{i}\right)=\left(\left(m_{2} u_{2} d_{1}^{3}+m_{1} u_{1} d_{2}^{3}\right)\right. \\
& \left.+d_{1} d_{2}\left(\left(m_{1} u_{1} d_{1}+m_{2} u_{2} d_{2}\right) \frac{\theta_{3}^{2}+\theta_{4}^{2}}{\theta_{3}^{2}}+\left(m_{2} u_{2} d_{1}+m_{1} u_{1} d_{2}\right) \frac{\theta_{3}^{4}+\theta_{2}^{4}+\theta_{3}^{2} \theta_{4}^{2}}{\theta_{3}^{2} \theta_{2}^{2}}\right)\right) \frac{\theta_{3}^{4}}{\theta_{4}^{2} \theta_{2}^{2}}, \\
& H_{3}^{(1)}\left(m_{i}, d_{i}\right)=\left(\left(m_{1} d_{1}^{4}+m_{2} d_{2}^{4}\right)\right. \\
& \left.+d_{1} d_{2}\left(\left(m_{1} d_{1}^{2}+m_{2} d_{2}^{2}\right) \frac{2 \theta_{3}^{4}+\theta_{2}^{4}}{\theta_{2}^{2} \theta_{3}^{2}}+\left(m_{2} d_{1}^{2}+m_{1} d_{2}^{2}\right) \frac{\theta_{2}^{2}}{\theta_{3}^{2}}\right)+3\left(m_{1}+m_{2}\right) d_{1}^{2} d_{2}^{2}\right) \frac{\theta_{3}^{4}}{\theta_{4}^{4}}, \\
& H_{3}^{(2)}\left(m_{i}, d_{i}\right)=\left(\left(m_{2} d_{1}^{4}+m_{1} d_{2}^{4}\right)\right. \\
& \left.+d_{1} d_{2}\left(\left(m_{1} d_{1}^{2}+m_{2} d_{2}^{2}\right) \frac{\theta_{2}^{2}}{\theta_{3}^{2}}+\left(m_{2} d_{1}^{2}+m_{1} d_{2}^{2}\right) \frac{2 \theta_{3}^{4}+\theta_{2}^{4}}{\theta_{2}^{2} \theta_{3}^{2}}\right)+3\left(m_{1}+m_{2}\right) d_{1}^{2} d_{2}^{2}\right) \frac{\theta_{3}^{2} \theta_{2}^{4}}{\theta_{4}^{6}}, \\
& H_{4}^{(1)}\left(m_{i}, d_{i}\right)=\left(\left(m_{1}^{2} d_{1}^{4}+m_{2}^{2} d_{2}^{4}\right)-2 d_{1} d_{2}\left(m_{2}^{2} d_{1}^{2}+m_{1}^{2} d_{2}^{2}\right) \frac{\theta_{2}^{2}}{\theta_{3}^{2}}-\left(m_{1}^{2}+m_{2}^{2}\right) d_{1}^{2} d_{2}^{2} \frac{2 \theta_{3}^{4}+\theta_{2}^{4}}{\theta_{3}^{4}}\right) \frac{\theta_{3}^{4}}{\theta_{4}^{4}}, \\
& H_{4}^{(2)}\left(m_{i}, d_{i}\right)=\left(\left(m_{2}^{2} d_{1}^{4}+m_{1}^{2} d_{2}^{4}\right)+2 d_{1} d_{2}\left(m_{2}^{2} d_{1}^{2}+m_{1}^{2} d_{2}^{2}\right) \frac{\theta_{2}^{2}}{\theta_{3}^{2}}+\left(m_{1}^{2}+m_{2}^{2}\right) d_{1}^{2} d_{2}^{2} \frac{\theta_{2}^{4}}{\theta_{3}^{4}}\right) \frac{\theta_{3}^{4}}{\theta_{4}^{4}}, \\
& I_{4}^{(1)}\left(u_{i}, d_{i}\right)=\left(u_{1} d_{1}^{5}+u_{2} d_{2}^{5}+d_{1} d_{2}\left(u_{2} d_{1}^{3}+u_{1} d_{2}^{3}\right) \frac{-3 \theta_{2}^{8}+2 \theta_{4}^{6}\left(\theta_{3}^{2}-\theta_{4}^{2}\right)+\theta_{4}^{2} \theta_{2}^{4}\left(4 \theta_{3}^{2}-5 \theta_{4}^{2}\right)}{\theta_{3}^{4} \theta_{2}^{4}}\right. \\
& +d_{1}^{2} d_{2}^{2}\left(\left(u_{2} d_{1}+u_{1} d_{2}\right) \frac{-8 \theta_{2}^{8}\left(\theta_{3}^{2}-\theta_{4}^{2}\right)-2 \theta_{4}^{8}\left(\theta_{3}^{2}-\theta_{4}^{2}\right)-\theta_{4}^{4} \theta_{2}^{4}\left(8 \theta_{3}^{2}-9 \theta_{4}^{2}\right)}{\theta_{3}^{4} \theta_{2}^{6}}\right. \\
& \left.\left.+\left(u_{1} d_{1}+u_{2} d_{2}\right) \frac{-6 \theta_{2}^{8}+4 \theta_{4}^{2} \theta_{2}^{4}\left(\theta_{3}^{2}-2 \theta_{4}^{2}\right)+3 \theta_{4}^{6}\left(\theta_{3}^{2}-\theta_{4}^{2}\right)}{\theta_{3}^{4} \theta_{2}^{4}}\right)\right) \frac{\theta_{3}^{5}}{\theta_{4}^{5}}, \\
& I_{4}^{(2)}\left(u_{i}, d_{i}\right)=\left(u_{2} d_{1}^{5}+u_{1} d_{2}^{5}+d_{1} d_{2}\left(u_{2} d_{1}^{3}+u_{1} d_{2}^{3}\right) \frac{\theta_{4}^{4}\left(\theta_{3}^{2}+\theta_{4}^{2}\right)+\theta_{2}^{4}\left(3 \theta_{3}^{2}+\theta_{4}^{2}\right)}{\theta_{3}^{4} \theta_{2}^{2}}\right. \\
& \left.+d_{1}^{2} d_{2}^{2}\left(\left(u_{1} d_{1}+u_{2} d_{2}\right) \frac{\theta_{2}^{2}\left(\theta_{3}^{2}+\theta_{4}^{2}\right)}{\theta_{3}^{4}}+\left(u_{2} d_{1}+u_{1} d_{2}\right) \frac{3 \theta_{2}^{4}+2 \theta_{4}^{2}\left(\theta_{3}^{2}+\theta_{4}^{2}\right)}{\theta_{3}^{4}}\right)\right) \frac{\theta_{3}^{4} \theta_{2}^{4}}{\theta_{4}^{6}}, \\
& I_{4}^{(3)}\left(u_{i}, d_{i}\right)=\left(d_{1} d_{2}\left(u_{1} d_{1}^{3}+u_{2} d_{2}^{3}\right)+d_{1}^{2} d_{2}^{2}\left(\left(u_{1} d_{1}+u_{2} d_{2}\right) \frac{\theta_{4}^{4}\left(2 \theta_{3}^{2}-\theta_{4}^{2}\right)+\theta_{2}^{4}\left(3 \theta_{3}^{2}-\theta_{4}^{2}\right)}{\theta_{3}^{4} \theta_{2}^{2}}\right.\right. \\
& \left.\left.+\left(u_{2} d_{1}+u_{1} d_{2}\right) \frac{3 \theta_{2}^{8}-\theta_{4}^{6}\left(\theta_{3}^{2}-\theta_{4}^{2}\right)-\theta_{4}^{2} \theta_{2}^{4}\left(2 \theta_{3}^{2}+4 \theta_{4}^{2}\right)}{\theta_{3}^{4} \theta_{2}^{4}}\right)\right) \frac{\theta_{3}^{5}}{\theta_{4}^{3} \theta_{2}^{2}}, \\
& J_{4}^{(1)}\left(m_{i}, d_{i}\right)=\left(\left(m_{1} d_{1}^{6}+m_{2} d_{2}^{6}\right)+3 d_{1} d_{2}\left(m_{1} d_{1}^{4}+m_{2} d_{2}^{4}\right) \frac{\theta_{2}^{2}}{\theta_{3}^{2}}\right. \\
& \left.+3 d_{1}^{2} d_{2}^{2}\left(m_{1} d_{1}^{2}+m_{2} d_{2}^{2}\right) \frac{\theta_{2}^{4}}{\theta_{3}^{4}}+\left(m_{1}+m_{2}\right) d_{1}^{3} d_{2}^{3} \frac{\theta_{2}^{6}}{\theta_{3}^{6}}\right) \frac{\theta_{3}^{6}}{\theta_{4}^{6}}, \\
& J_{4}^{(2)}\left(m_{i}, d_{i}\right)=\left(\left(m_{2} d_{1}^{6}+m_{1} d_{2}^{6}\right)+3 d_{1} d_{2}\left(m_{2} d_{1}^{4}+m_{1} d_{2}^{4}\right) \frac{\theta_{2}^{2}}{\theta_{3}^{2}}\right. \\
& \left.+3 d_{1}^{2} d_{2}^{2}\left(m_{2} d_{1}^{2}+m_{1} d_{2}^{2}\right) \frac{\theta_{2}^{4}}{\theta_{3}^{4}}+\left(m_{1}+m_{2}\right) d_{1}^{3} d_{2}^{3} \frac{\theta_{2}^{6}}{\theta_{3}^{6}}\right) \frac{\theta_{3}^{4} \theta_{2}^{4}}{\theta_{4}^{8}},
\end{aligned}
$$




$$
\begin{aligned}
J_{4}^{(3)}\left(m_{i}, d_{i}\right)=\left(d_{1} d_{2}\left(m_{1} d_{1}^{4}+m_{2} d_{2}^{4}\right)\right. & d_{1}^{2} d_{2}^{2}\left(\left(m_{1} d_{1}^{2}+m_{2} d_{2}^{2}\right) \frac{\theta_{3}^{4}+2 \theta_{2}^{4}}{\theta_{3}^{2} \theta_{2}^{2}}+\left(m_{2} d_{1}^{2}+m_{1} d_{2}^{2}\right) \frac{\theta_{2}^{2}}{\theta_{3}^{2}}\right) \\
& \left.+\left(m_{1}+m_{2}\right) d_{1}^{3} d_{2}^{3} \frac{\theta_{3}^{8}+\theta_{2}^{8}+\theta_{4}^{4} \theta_{2}^{4}}{\theta_{3}^{8}}\right) \frac{\theta_{3}^{6}}{\theta_{4}^{4} \theta_{2}^{2}}, \\
J_{4}^{(4)}\left(m_{i}, d_{i}\right)=\left(d_{1} d_{2}\left(m_{2} d_{1}^{4}+m_{1} d_{2}^{4}\right)\right. & +d_{1}^{2} d_{2}^{2}\left(\left(m_{1} d_{1}^{2}+m_{2} d_{2}^{2}\right) \frac{\theta_{2}^{2}}{\theta_{3}^{2}}+\left(m_{2} d_{1}^{2}+m_{1} d_{2}^{2}\right) \frac{\theta_{3}^{4}+2 \theta_{2}^{4}}{\theta_{3}^{2} \theta_{2}^{2}}\right) \\
& \left.+\left(m_{1}+m_{2}\right) d_{1}^{3} d_{2}^{3} \frac{\theta_{3}^{8}+\theta_{2}^{8}+\theta_{4}^{4} \theta_{2}^{4}}{\theta_{3}^{8}}\right) \frac{\theta_{3}^{4} \theta_{2}^{2}}{\theta_{4}^{6}}
\end{aligned}
$$

where each block is invariant under the $T$ transformation (5.45) and the permutation symmetry, and their subscripts coincide with their mass dimensions.

Open Access. This article is distributed under the terms of the Creative Commons Attribution License (CC-BY 4.0), which permits any use, distribution and reproduction in any medium, provided the original author(s) and source are credited.

\section{References}

[1] N.A. Nekrasov, Seiberg-Witten prepotential from instanton counting, Adv. Theor. Math. Phys. 7 (2003) 831 [hep-th/0206161] [INSPIRE].

[2] R. Flume and R. Poghossian, An Algorithm for the microscopic evaluation of the coefficients of the Seiberg-Witten prepotential, Int. J. Mod. Phys. A 18 (2003) 2541 [hep-th/0208176] [INSPIRE].

[3] U. Bruzzo, F. Fucito, J.F. Morales and A. Tanzini, Multiinstanton calculus and equivariant cohomology, JHEP 05 (2003) 054 [hep-th/0211108] [INSPIRE].

[4] N. Nekrasov and A. Okounkov, Seiberg-Witten theory and random partitions, Prog. Math. 244 (2006) 525 [hep-th/0306238] [INSPIRE].

[5] H. Nakajima and K. Yoshioka, Lectures on instanton counting, in CRM Workshop on Algebraic Structures and Moduli Spaces, (2003) [math/0311058] [INSPIRE].

[6] M. Buican, S. Giacomelli, T. Nishinaka and C. Papageorgakis, Argyres-Douglas Theories and S-duality, JHEP 02 (2015) 185 [arXiv: 1411.6026] [InSPIRE].

[7] M. Del Zotto, C. Vafa and D. Xie, Geometric engineering, mirror symmetry and $6 \mathrm{~d}_{(1,0)} \rightarrow 4 \mathrm{~d}_{(\mathcal{N}=2)}$, JHEP 11 (2015) 123 [arXiv: 1504.08348] [INSPIRE].

[8] S. Cecotti and M. Del Zotto, Higher S-dualities and Shephard-Todd groups, JHEP 09 (2015) 035 [arXiv: 1507.01799] [INSPIRE].

[9] D. Xie and S.-T. Yau, New $N=2$ dualities, arXiv:1602.03529 [INSPIRE].

[10] D. Xie and S.-T. Yau, Argyres-Douglas matter and $N=2$ dualities, arXiv:1701.01123 [INSPIRE].

[11] M. Buican, Z. Laczko and T. Nishinaka, $\mathcal{N}=2$ S-duality revisited, JHEP 09 (2017) 087 [arXiv:1706.03797] [INSPIRE].

[12] D. Xie and K. Ye, Argyres-Douglas matter and S-duality: Part II, JHEP 03 (2018) 186 [arXiv: 1711.06684] [INSPIRE].

[13] M. Buican, Z. Laczko and T. Nishinaka, Flowing from 16 to 32 Supercharges, JHEP 10 (2018) 175 [arXiv: 1807.02785] [INSPIRE]. 
[14] P.C. Argyres and M.R. Douglas, New phenomena in SU(3) supersymmetric gauge theory, Nucl. Phys. B 448 (1995) 93 [hep-th/9505062] [InSPIRE].

[15] P.C. Argyres, M.R. Plesser, N. Seiberg and E. Witten, New $N=2$ superconformal field theories in four-dimensions, Nucl. Phys. B 461 (1996) 71 [hep-th/9511154] [INSPIRE].

[16] T. Eguchi, K. Hori, K. Ito and S.-K. Yang, Study of $N=2$ superconformal field theories in four-dimensions, Nucl. Phys. B 471 (1996) 430 [hep-th/9603002] [INSPIRE].

[17] G. Bonelli, K. Maruyoshi and A. Tanzini, Wild Quiver Gauge Theories, JHEP 02 (2012) 031 [arXiv:1112.1691] [INSPIRE].

[18] D. Gaiotto and J. Teschner, Irregular singularities in Liouville theory and Argyres-Douglas type gauge theories, I, JHEP 12 (2012) 050 [arXiv:1203.1052] [INSPIRE].

[19] L.F. Alday, D. Gaiotto and Y. Tachikawa, Liouville Correlation Functions from Four-dimensional Gauge Theories, Lett. Math. Phys. 91 (2010) 167 [arXiv:0906.3219] [INSPIRE].

[20] D. Gaiotto, Asymptotically free $\mathcal{N}=2$ theories and irregular conformal blocks, J. Phys. Conf. Ser. 462 (2013) 012014 [arXiv: 0908. 0307] [INSPIRE].

[21] H. Kanno and M. Taki, Generalized Whittaker states for instanton counting with fundamental hypermultiplets, JHEP 05 (2012) 052 [arXiv: 1203.1427] [INSPIRE].

[22] T. Nishinaka and C. Rim, Matrix models for irregular conformal blocks and Argyres-Douglas theories, JHEP 10 (2012) 138 [arXiv:1207.4480] [INSPIRE].

[23] C. Rim, Irregular conformal block and its matrix model, arXiv:1210.7925 [INSPIRE].

[24] H. Kanno, K. Maruyoshi, S. Shiba and M. Taki, $\mathcal{W}_{3}$ irregular states and isolated $\mathcal{N}=2$ superconformal field theories, JHEP 03 (2013) 147 [arXiv:1301.0721] [INSPIRE].

[25] Y. Matsuo, C. Rim and H. Zhang, Construction of Gaiotto states with fundamental multiplets through Degenerate DAHA, JHEP 09 (2014) 028 [arXiv:1405.3141] [INSPIRE].

[26] S.K. Choi, C. Rim and H. Zhang, Irregular conformal block, spectral curve and flow equations, JHEP 03 (2016) 118 [arXiv: 1510.09060] [INSPIRE].

[27] C. Rim and H. Zhang, Nekrasov and Argyres-Douglas theories in spherical Hecke algebra representation, Nucl. Phys. B 919 (2017) 182 [arXiv:1608.05027] [INSPIRE].

[28] H. Itoyama, T. Oota and K. Yano, Discrete Painleve system and the double scaling limit of the matrix model for irregular conformal block and gauge theory, Phys. Lett. B 789 (2019) 605 [arXiv: 1805. 05057] [INSPIRE].

[29] H. Itoyama, T. Oota and K. Yano, Discrete Painlevé system for the partition function of $N_{f}=2 \mathrm{SU}(2)$ supersymmetric gauge theory and its double scaling limit, J. Phys. A $\mathbf{5 2}$ (2019) 415401 [arXiv: 1812.00811] [INSPIRE].

[30] T. Nishinaka and T. Uetoko, Argyres-Douglas theories and Liouville Irregular States, JHEP 09 (2019) 104 [arXiv: 1905.03795] [INSPIRE].

[31] D. Xie, General Argyres-Douglas Theory, JHEP 01 (2013) 100 [arXiv:1204.2270] [INSPIRE].

[32] C. Beem and W. Peelaers, Argyres-Douglas Theories in Class S Without Irregularity, arXiv:2005.12282 [INSPIRE].

[33] N. Wyllard, $A_{N-1}$ conformal Toda field theory correlation functions from conformal $N=2$ $\mathrm{SU}(N)$ quiver gauge theories, JHEP 11 (2009) 002 [arXiv:0907.2189] [INSPIRE]. 
[34] V.A. Alba, V.A. Fateev, A.V. Litvinov and G.M. Tarnopolskiy, On combinatorial expansion of the conformal blocks arising from AGT conjecture, Lett. Math. Phys. 98 (2011) 33 [arXiv: 1012.1312] [INSPIRE].

[35] M. Buican, L. Li and T. Nishinaka, Peculiar Index Relations, 2D TQFT, and Universality of SUSY Enhancement, JHEP 01 (2020) 187 [arXiv:1907.01579] [INSPIRE].

[36] T.W. Grimm, A. Klemm, M. Mariño and M. Weiss, Direct Integration of the Topological String, JHEP 08 (2007) 058 [hep-th/0702187] [INSPIRE].

[37] N.A. Nekrasov and S.L. Shatashvili, Quantization of Integrable Systems and Four Dimensional Gauge Theories, in 16th International Congress on Mathematical Physics, (2009) [DOI] [arXiv:0908.4052] [INSPIRE].

[38] K. Ito, S. Kanno and T. Okubo, Quantum periods and prepotential in $\mathcal{N}=2 \mathrm{SU}(2) S Q C D$, JHEP 08 (2017) 065 [arXiv:1705.09120] [INSPIRE].

[39] K. Ito and T. Okubo, Quantum periods for $\mathcal{N}=2 \mathrm{SU}(2) S Q C D$ around the superconformal point, Nucl. Phys. B 934 (2018) 356 [arXiv:1804.04815] [INSPIRE].

[40] K. Ito, S. Koizumi and T. Okubo, Quantum Seiberg-Witten curve and Universality in Argyres-Douglas theories, Phys. Lett. B 792 (2019) 29 [arXiv:1903.00168] [inSPIRE].

[41] K. Ito, S. Koizumi and T. Okubo, Quantum Seiberg-Witten periods for $\mathcal{N}=2 \mathrm{SU}\left(N_{c}\right) S Q C D$ around the superconformal point, Nucl. Phys. B 954 (2020) 115004 [arXiv:2001.08891] [INSPIRE].

[42] H. Awata and Y. Yamada, Five-dimensional AGT Conjecture and the Deformed Virasoro Algebra, JHEP 01 (2010) 125 [arXiv:0910.4431] [INSPIRE].

[43] H. Awata and Y. Yamada, Five-dimensional AGT Relation and the Deformed beta-ensemble, Prog. Theor. Phys. 124 (2010) 227 [arXiv:1004.5122] [INSPIRE].

[44] S. Yanagida, Five-dimensional SU(2) AGT conjecture and recursive formula of deformed Gaiotto state, J. Math. Phys. 51 (2010) 123506 [arXiv:1005.0216] [InSPIRE].

[45] M. Taki, On AGT-W Conjecture and q-Deformed W-Algebra, arXiv:1403.7016 [INSPIRE].

[46] V. Mitev and E. Pomoni, Toda 3-Point Functions From Topological Strings, JHEP 06 (2015) 049 [arXiv: 1409.6313] [INSPIRE].

[47] M. Isachenkov, V. Mitev and E. Pomoni, Toda 3-Point Functions From Topological Strings II, JHEP 08 (2016) 066 [arXiv: 1412.3395] [INSPIRE].

[48] H. Awata, H. Fujino and Y. Ohkubo, Crystallization of deformed Virasoro algebra, Ding-Iohara-Miki algebra and 5D AGT correspondence, J. Math. Phys. 58 (2017) 071704 [arXiv: 1512.08016] [INSPIRE].

[49] J.-E. Bourgine, M. Fukuda, Y. Matsuo, H. Zhang and R.-D. Zhu, Coherent states in quantum $\mathcal{W}_{1+\infty}$ algebra and qq-character for 5d Super Yang-Mills, PTEP 2016 (2016) 123B05 [arXiv: 1606.08020] [INSPIRE].

[50] S. Pasquetti, Holomorphic blocks and the 5d AGT correspondence, J. Phys. A 50 (2017) 443016 [arXiv: 1608.02968] [INSPIRE].

[51] A. Negut, The q-AGT-W relations via shuffle algebras, Commun. Math. Phys. 358 (2018) 101 [arXiv: 1608.08613] [INSPIRE].

[52] H. Hayashi and K. Ohmori, 5d/6d DE instantons from trivalent gluing of web diagrams, JHEP 06 (2017) 078 [arXiv:1702.07263] [INSPIRE]. 
[53] S. Kanno, Y. Matsuo and S. Shiba, $W(1+\infty)$ algebra as a symmetry behind AGT relation, Phys. Rev. D 84 (2011) 026007 [arXiv: 1105.1667] [INSPIRE].

[54] H. Awata, B. Feigin, A. Hoshino, M. Kanai, J. Shiraishi and S. Yanagida, Notes on Ding-Iohara algebra and AGT conjecture, arXiv:1106.4088 [INSPIRE].

[55] S. Kanno, Y. Matsuo and H. Zhang, Extended Conformal Symmetry and Recursion Formulae for Nekrasov Partition Function, JHEP 08 (2013) 028 [arXiv:1306.1523] [INSPIRE].

[56] J.-E. Bourgine, Y. Matsuo and H. Zhang, Holomorphic field realization of $S H^{c}$ and quantum geometry of quiver gauge theories, JHEP 04 (2016) 167 [arXiv:1512.02492] [INSPIRE].

[57] A. Mironov, A. Morozov and Y. Zenkevich, Ding-Iohara-Miki symmetry of network matrix models, Phys. Lett. B 762 (2016) 196 [arXiv:1603.05467] [InSPIRE].

[58] H. Awata et al., Explicit examples of DIM constraints for network matrix models, JHEP 07 (2016) 103 [arXiv : 1604.08366] [INSPIRE].

[59] H. Awata et al., Toric Calabi-Yau threefolds as quantum integrable systems. $\mathcal{R}$-matrix and $\mathcal{R} \mathcal{T} \mathcal{T}$ relations, JHEP 10 (2016) 047 [arXiv: 1608.05351] [INSPIRE].

[60] H. Awata et al., Anomaly in RTT relation for DIM algebra and network matrix models, Nucl. Phys. B 918 (2017) 358 [arXiv: 1611.07304] [INSPIRE].

[61] H. Awata et al., Generalized Knizhnik-Zamolodchikov equation for Ding-Iohara-Miki algebra, Phys. Rev. D 96 (2017) 026021 [arXiv:1703.06084] [InSPIRE].

[62] J.-E. Bourgine, M. Fukuda, K. Harada, Y. Matsuo and R.-D. Zhu, $(p, q)$-webs of DIM representations, $5 d \mathcal{N}=1$ instanton partition functions and qq-characters, JHEP 11 (2017) 034 [arXiv: 1703.10759] [INSPIRE].

[63] J.-E. Bourgine, M. Fukuda, Y. Matsuo and R.-D. Zhu, Reflection states in Ding-Iohara-Miki algebra and brane-web for D-type quiver, JHEP 12 (2017) 015 [arXiv:1709.01954] [INSPIRE].

[64] H. Awata, H. Kanno, A. Mironov, A. Morozov, K. Suetake and Y. Zenkevich, $(q, t)-K Z$ equations for quantum toroidal algebra and Nekrasov partition functions on ALE spaces, JHEP 03 (2018) 192 [arXiv:1712.08016] [INSPIRE].

[65] S. Sasa, A. Watanabe and Y. Matsuo, A note on the S-dual basis in the free fermion system, PTEP 2020 (2020) 023B02 [arXiv: 1904.04766] [INSPIRE].

[66] M. Buican and T. Nishinaka, $\mathcal{N}=4$ SYM, Argyres-Douglas Theories, and an Exact Graded Vector Space Isomorphism, arXiv:2012.13209 [INSPIRE].

[67] C. Beem, M. Lemos, P. Liendo, W. Peelaers, L. Rastelli and B.C. van Rees, Infinite Chiral Symmetry in Four Dimensions, Commun. Math. Phys. 336 (2015) 1359 [arXiv:1312.5344] [INSPIRE].

[68] K. Ito and H. Shu, ODE/IM correspondence and the Argyres-Douglas theory, JHEP 08 (2017) 071 [arXiv: 1707.03596] [INSPIRE].

[69] L. Hollands, C.A. Keller and J. Song, From SO/Sp instantons to W-algebra blocks, JHEP 03 (2011) 053 [arXiv: 1012.4468] [INSPIRE].

[70] L. Hollands, C.A. Keller and J. Song, Towards a $4 d / 2 d$ correspondence for Sicilian quivers, JHEP 10 (2011) 100 [arXiv:1107.0973] [InSPIRE].

[71] F. Fucito, J.F. Morales and R. Poghossian, Instantons on quivers and orientifolds, JHEP 10 (2004) 037 [hep-th/0408090] [INSPIRE]. 